\title{
Réflexions multiples. Le miroir et ses usages rituels en Afrique centrale
}

Julien Bonhomme

\section{(2) OpenEdition}

1 Journals

Édition électronique

URL : https://journals.openedition.org/imagesrevues/147

DOI : 10.4000/imagesrevues. 147

ISSN : 1778-3801

Éditeur :

Centre d'Histoire et Théorie des Arts, Groupe d'Anthropologie Historique de l'Occident Médiéval, Laboratoire d'Anthropologie Sociale, UMR 8210 Anthropologie et Histoire des Mondes Antiques

Référence électronique

Julien Bonhomme, « Réflexions multiples. Le miroir et ses usages rituels en Afrique centrale », Images Re-vues [En ligne], 4 | 2007, document 9, mis en ligne le 01 janvier 2007, consulté le 08 décembre 2022. URL : http://journals.openedition.org/imagesrevues/147 ; DOI : https://doi.org/10.4000/ imagesrevues. 147

Ce document a été généré automatiquement le 30 janvier 2021.

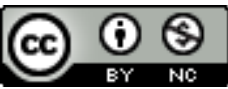

Creative Commons - Attribution - Pas d'Utilisation Commerciale 4.0 International - CC BY-NC 4.0 https://creativecommons.org/licenses/by-nc/4.0/ 


\title{
Réflexions multiples. Le miroir et ses usages rituels en Afrique centrale
}

\author{
Julien Bonhomme
}

«Bioy Casarès se rappela alors qu'un des hérésiarques d'Uqbar avait déclaré que les miroirs et la copulation étaient abominables parce qu'ils multipliaient le nombre des hommes ».

(J.L. Borges, « Tlön Uqbar Orbis Tertius » in Fictions, Paris, Gallimard, 1951) 
1 On connaît la fortune du miroir dans la littérature occidentale: des contes traditionnels (Blanche-Neige) aux contes modernes (Lewis Carroll, De l'autre côté du miroir et ce qu'Alice y trouva, 1896), du romantisme fantastique (E.T.A. Hoffmann, L'histoire du reflet perdu, 1815, Guy de Maupassant, Le Horla, 1887) au surréalisme (Jacques Rigaut, Lord Patchogue, 1924, Jean

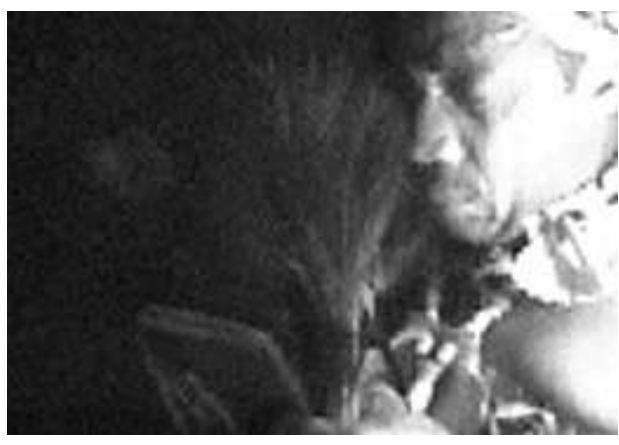
Cocteau, Le Sang d'un Poète, 1930). Si le miroir excite autant les imaginations, c'est qu'il est un objet étrange. Cette étrangeté provient du double paradoxe de la perception spéculaire: d'une part, le reflet de soi dans le miroir dédouble le sujet; d'autre part, l'espace du reflet est perçu comme le prolongement de l'espace réel audelà du miroir. Le double spéculaire ouvre sur des vertiges identitaires, l'espace spéculaire sur des vertiges ontologiques. D'un côté, c'est Lord Patchogue qui se cherche et se perd dans une série de reflets. De l'autre, c'est Alice qui découvre un monde fantastique en passant à travers le miroir. L'imaginaire littéraire du miroir s'appuie ainsi sur l'étrangeté bien réelle d'une expérience perceptive qui donne à voir un double de soi et du monde au statut problématique. Cet étonnement face au miroir se retrouve jusque dans l'étymologie du terme qui vient du latin mirari, "admirer " mais aussi " regarder avec étonnement», "être surpris ${ }^{1}$. Si J. Goody a souligné l'ambivalence cognitive généralement suscitée par les images, l'image spéculaire recèle une ambivalence encore plus saillante ${ }^{2}$.

2 L'expérience des vertiges du miroir n'est toutefois pas un apanage occidental. D'une part, les miroirs sont présents depuis fort longtemps dans bien des parties du monde, en Chine ou au Japon comme en Égypte ancienne ${ }^{3}$. D'autre part, la surface réfléchissante de l'eau (ou d'un minéral cristallin) a tout de même permis aux peuples sans miroir de ne pas rester des peuples sans reflet, avant que le commerce ne diffuse universellement les petits miroirs à main. En Afrique centrale, cela fait cinq siècles que les miroirs européens ont commencé à circuler le long des voies de traite. Tout un ensemble de représentations et de pratiques les concernant a donc eu le temps de s'y développer et de s'y stabiliser: représentations inquiétantes qui associent miroirs, fantômes et sorciers, mais aussi pratiques divinatoires et initiatiques où le miroir joue un rôle de premier plan. Ces représentations et ces pratiques liées au miroir en Afrique centrale sont l'objet du présent article.

3 Ce travail s'inscrit dans une anthropologie attentive aux objets, à leurs propriétés physiques comme à la diversité socioculturelle de leurs usages. Il s'agit ainsi d'étudier la diffusion d'un élément de la culture matérielle occidentale dans des sociétés étrangères: quelles réappropriations ont accompagné la diffusion des miroirs en Afrique centrale? Ceci implique également de dépasser le cadre de l'ethnographie africaine par une mise en perspective comparatiste sur la place du miroir dans d'autres civilisations et en d'autres temps. Mais cela ne signifie nullement négliger les propriétés matérielles des objets au profit exclusif de leur symbolisme culturel. L'objet ne doit donc pas être envisagé in abstracto mais toujours replacé dans des contextes d'usage singuliers, ce qui suppose de privilégier l'étude des pratiques sur celle des représentations. Il s'agit de dégager moins une symbolique du miroir qu'une 
pragmatique des manipulations spéculaires : les miroirs ne sont pas seulement « bons à penser ", ils sont également " bons à manipuler ». S'ils occupent une telle place dans la divination et l'initiation en Afrique centrale, c'est en effet qu'ils ont été sélectionnés pour des propriétés optiques précises qui vont pouvoir être mises en jeu dans des dispositifs rituels aussi concrets qu'efficaces. Nous verrons ainsi que le miroir constitue un instrument de choix pour opérer des manipulations rituelles paradoxales. Son pouvoir réfléchissant permet une mise en abyme du sujet, tandis que sa manipulation permet une mise en rapport du sujet avec une série d'autres personnes. Le miroir rend ainsi possible un travail complexe sur l'identité à travers une mise en relation ambiguë du soi et de l'autre.

4 La première partie de l'article analyse les représentations associant miroir et fantômes en Afrique centrale. Les deuxième et troisième parties sont consacrées aux usages rituels (divinatoires et initiatiques) du miroir en Afrique centrale, et notamment au Gabon. La dernière partie met en perspective le rôle instrumental du miroir pour des conceptions analogiques du sujet, avant de conclure sur l'importance des mises en scène spéculaires dans la « rencontre coloniale » entre l'Afrique et l'Europe ${ }^{4}$.

\section{Miroir spectral : réfléchir les fantômes}

$5 \quad$ Les côtes atlantiques de l'Afrique centrale sont atteintes par les navigateurs portugais dans le dernier tiers du XV ${ }^{e}$ siècle. Les miroirs font alors très tôt partie du commerce de traite et sont particulièrement recherchés par les autochtones: 0 . Dapper note l'importance des « miroirs à quadre noir » dans le Loango du XVII ${ }^{e}$ siècle ${ }^{5}$. Au XIX ${ }^{e}$ siècle, les miroirs font toujours partie du lot de marchandises des factoreries ${ }^{6}$. Au fur et à mesure qu'ils se diffusent de la côte vers l'hinterland, les petits miroirs à main remplacent ainsi les coupes d'eau ou les réflecteurs en métal local ou en cristal de roche - leur plus grande maniabilité et leur meilleur pouvoir réfléchissant leur assurant un succès considérable. Objets recherchés, les miroirs sont pourtant également des objets dangereux, associés aux fantômes et aux sorciers. En Afrique centrale, circulent en effet aujourd'hui un ensemble de représentations autour des pouvoirs inquiétants du miroir. Au Gabon et au Congo, on retourne les glaces dans la maison d'un mort, de même qu'on ne regarde pas dans le rétroviseur d'un corbillard, de peur d'être tourmenté par le fantôme du défunt et de mourir soi-même ${ }^{7}$. Il ne faut pas se regarder la nuit dans un miroir de peur d'y être happé par des fantômes. Au Nord Gabon, la sorcellerie du Kong userait d'une boîte sertie de miroirs: lorsque le visage de l'envoûté y apparait, ce dernier, désormais captif de l'image spéculaire, se trouve transformé en zombie servile au service du sorcier. Non seulement le miroir reflète les fantômes, mais il menace de transformer le sujet lui-même en fantôme. Comme J. Tonda le relève, le miroir «spectralise » la personne. Il est toujours un «miroir anormal» qui donne à voir la troublante image d'un sujet défiguré par des pouvoirs occultes qui le dépassent (la mort, la sorcellerie).

6 Cette association menaçante entre miroir et mort se retrouve dans le folklore européen ${ }^{8}$. Il faut voiler les glaces dans la maison d'un mort, de peur que l'âme du défunt ne reste dans le foyer ou que celui qui s'y mire n'y perde la sienne ou ne meure. Il est pareillement défendu d'exposer le cadavre devant un miroir, son reflet annonçant un second décès. Briser un miroir entraîne au pire la mort, au mieux sept ans de malheur. Il ne faut pas se regarder la nuit dans un miroir. Une curieuse croyance 
française stipule qu'on peut se voir dans une glace tel qu'on sera à l'heure de sa mort, si on accomplit un certain rituel pendant la nuit de l'Épiphanie. Ce pouvoir inquiétant des glaces à refléter les fantômes ou à provoquer la mort voisine avec de probables usages du miroir dans la sorcellerie européenne. Jusqu'au XVIII siècle, on appelle «miroir des sorcières", ou simplement "sorcière", un miroir rond et bosselé qui fait voir d'étranges reflets.

7 Le miroir spectral d'Afrique centrale est-il alors un emprunt au folklore européen (les représentations se diffusant en même temps que leur support matériel) ? La réponse, difficile à établir, n'a en réalité que peu d'importance. Le comparatisme "sans rivages " des folkloristes du début du $\mathrm{XX}^{\mathrm{e}}$ siècle a de toute façon montré que des représentations similaires se retrouvaient largement en d'autres temps et d'autres lieux. En Afrique centrale ou ailleurs, il semble bien que cette menace des fantômes spéculaires témoigne d'une vulnérabilité du sujet face au pouvoir captivant du miroir. La dangerosité supposée du miroir participe ainsi de ces "périls de l'âme» dont parle J. Frazer". La menace est d'autant plus grande qu'en Afrique centrale, l'esprit est pensé d'après un modèle optique : gedina-dina chez les mitsogo (Gabon central) ou nsisim chez les fang (nord Gabon, sud Cameroun) désigne l'esprit mais aussi l'ombre et le reflet. Cette représentation de l'âme-ombre ou de l'âme-reflet est extrêmement répandue en Afrique $^{10}$. Ombre et reflet sont donc des images particulièrement sensibles, puisque c'est l'intégrité du sujet qui s'y donne à voir et qui s'y joue. Par extension, ces périls de l'âme touchent également la photographie, comme en témoigne le fait qu'au Congo et au Gabon, le négatif photographique soit un "diable ${ }^{11}:$ il donne du sujet représenté une image inversée et spectrale.

Le lien intime du sujet à son esprit - fondement de son intégrité cognitive - est donc conçu sur le modèle de l'image. Cette analogie optique entraîne une certaine vulnérabilité et précarité du sujet. Ombre, reflet et image figurative relèvent en effet d'une expérience perceptive universellement déstabilisante, ce que montre bien la psychologie développementale ${ }^{12}$. Il faut presque trois ans à l'enfant pour acquérir la maturité cognitive nécessaire à la représentation adéquate des différents doubles visuels, même si une première étape clef est franchie autour de 18 mois avec l'identification gestuelle du reflet, de l'ombre et de la photographie. Avant cela, l'enfant ne sait pas exactement comment se comporter face à ces diverses représentations visuelles: il s'agit moins d'une confusion systématique entre l'image et le réel que d'une incertitude quant au statut de ces entités étranges. L'image de soi est plus troublante encore que toute autre image car l'incertitude ne porte pas seulement sur les catégories ontologiques mais aussi sur la frontière problématique du soi et de l'autre, ce que montre bien le cas du reflet. Devant un miroir, l'enfant commence par vouloir interagir comme avec un semblable (6-12 mois) avant de passer par une période ambivalente alternant fascination et évitement: son reflet lui paraît étrange et inquiétant. Ce «malaise spéculaire » précède de quelques mois la reconnaissance de soi (identification gestuelle puis verbale entre 18 et 36 mois). A côté de ce paradoxe du double spéculaire, l'enfant fait également l'expérience du paradoxe de l'espace spéculaire : l'espace du reflet est perçu comme le prolongement de l'espace réel (d'où les stratégies de contournement du miroir). Ce n'est qu'autour de trois ans que l'enfant aura résolu l'ensemble de ces équivoques face à un reflet désormais devenu familier. Cette familiarité est telle que la plupart des adultes cèdent à l'illusion que la connaissance de leur propre visage est une donnée immédiate de la perception : notre 
reflet facial nous semble fidèle alors que celui d'autrui nous paraît étrange (en raison de l'inversion énantiomorphe du reflet) ${ }^{13}$.

Avant de devenir une image évidente et fidèle, le reflet dans le miroir a donc été pour l'enfant une image troublante. Ce trouble spéculaire se retrouve dans certaines pathologies qui empêchent les malades de se reconnaître dans leur reflet ${ }^{14}$. Mais Freud rapporte une mésaventure personnelle (son reflet qu'il prend pour un étranger faisant intrusion dans sa cabine de train) illustrant que personne n'est à l'abri de l'« inquiétante étrangeté » - das Unheimliche - d'un double spéculaire qui peut surgir à la faveur d'une expérience fugace mais frappante ${ }^{15}$. Cette inquiétante étrangeté du reflet se retrouve dans les représentations évoquant des miroirs, où des fantômes apparaissent à la place d'un visage et où des vivants se font happer dans le monde des morts. Ces élaborations culturelles ne sont donc pas entièrement arbitraires, ayant pour caution l'étrangeté d'une expérience perceptive bien réelle. Il ne s'agit cependant évidemment pas de rapprocher le " primitif » et l'enfant, le « sauvage » et le fou comme a pu naguère le faire l'anthropologie évolutionniste. Les représentations africaines de miroirs spectraux tranchent nettement avec les usages quotidiens qu'hommes et femmes d'Afrique peuvent par ailleurs faire du miroir pour se raser, se coiffer ou se maquiller. Les contextes quotidiens reposent sur une appréhension parfaitement normale du miroir. Mais dans certains contextes spécifiques, les virtualités paradoxales du reflet spéculaire peuvent être systématiquement exploitées. Il s'agit de la mort et de la sorcellerie, événements impliquant justement une rupture de l'expérience quotidienne. Mais aussi, comme nous le verrons, de la divination et l'initiation, contextes rituels instaurant des cadres d'action et d'interprétation s'écartant des cadres ordinaires.

10 L'inquiétante étrangeté du miroir, fondée sur les paradoxes cognitifs du reflet, se focalise ainsi sur une altération de l'identité qui témoigne de la vulnérabilité du sujet. Le reflet donne une image précaire d'un sujet dont l'intégrité menace constamment de vaciller lorsque d'autres images viennent s'interposer à la place de la sienne: sous l'apparente évidence du visage familier transparaît la figure d'un spectre. Ces miroirs anormaux réfléchissent ainsi un " visage défiguré par d'étranges adversités ${ }^{16}$. Il s'agit en quelque sorte d'un anti-stade du miroir : l'« assomption jubilatoire» de l'enfant qui se reconnaît dans son reflet et passe d'une image morcelée de son corps à sa totalisation orthopédique ${ }^{17}$ se retourne en l'inquiétude d'un sujet qui ne se reconnaît plus dans son reflet et dont l'intégrité se diffracte dangereusement dans des images spectrales.

\section{Miroir divinatoire : réfléchir les sorciers}

11 A côté de ces miroirs spectraux, les miroirs sont également au centre de nombreuses pratiques rituelles en Afrique centrale. Ces usages rituels contrastent nettement avec les supposés usages maléfiques des miroirs, puisqu'ils ont justement pour fonction de déjouer les sorciers. Au Gabon, le miroir se trouve ainsi au principe d'un rite de protection domestique appelé «retour à l'envoyeur». Il s'agit de placer au seuil de l'habitation un fragment de miroir, bouclier réflecteur qui protège des fusils nocturnes et autres attaques mystiques en retournant l'agression contre l'agresseur. Le miroir réfléchissant permet ainsi de s'approprier la violence sorcière sans pour autant se compromettre avec elle - la violence agressive étant toujours attribuée à autrui. 
12 Mais c'est dans le champ de la divination que l'usage du miroir est le plus répandu. Les miroirs divinatoires se retrouvent en effet dans toute l'Afrique centrale, et plus largement en Afrique subsaharienne ${ }^{18}$. Là encore, il ne s'agit pas d'une spécificité africaine. En Mongolie, les chamanes se servent d'un réflecteur en bronze comme miroir divinatoire ou comme bouclier déflecteur contre les sorciers ${ }^{19}$. De même, en Amazonie, les chamanes waiãpi utilisent des miroirs pour refléter le monde des esprits $^{20}$. L'usage divinatoire du reflet est en outre attesté depuis l'Antiquité en Occident où il se décline dans toute une gamme de mantiques ${ }^{21}$ : catoptromancie (divination par le miroir), lécanomancie (coupe), gastromancie (fiole), onychomancie (ongle) ou cristallomancie. Au Moyen-Âge, les magiciens sont parfois appelés les «spéculaires ». Mais il n'est finalement pas étonnant que le miroir ait été tant utilisé comme support divinatoire en Afrique ou ailleurs étant donné ses propriétés optiques singulières.

Fig.1.

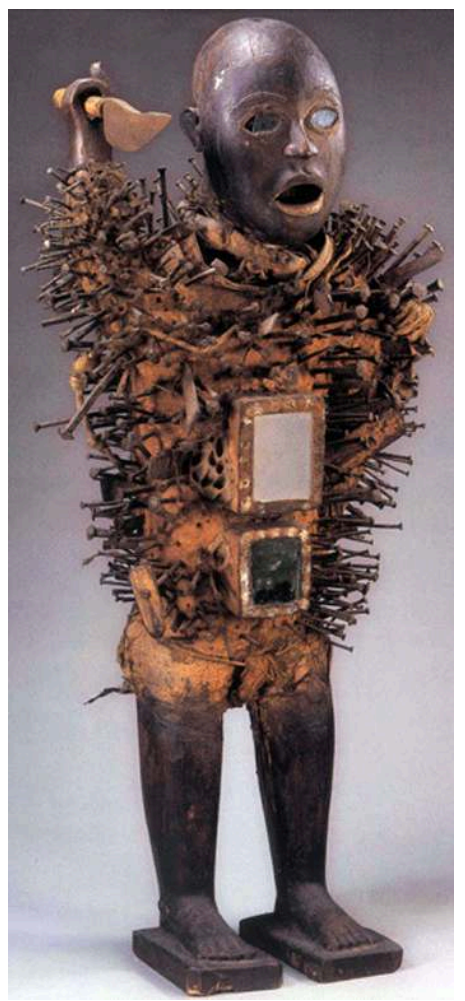

Fétiche nkisi nkondi (Angola). Musée Dapper. Dans Falgayrettes-Leveau, Christiane (dir.). Le Geste kôngo. Paris, Dapper, 2002, p. 45. 
Fig.2.

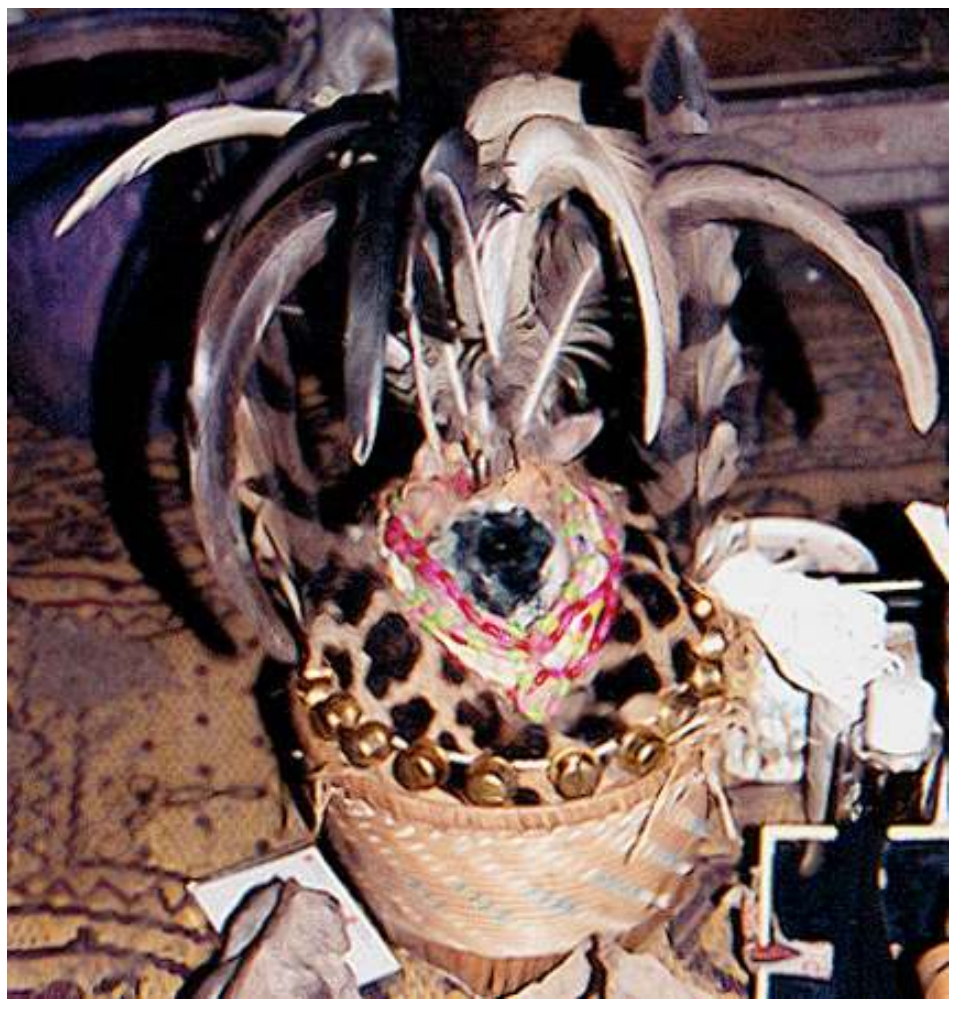

Fétiche du Bwete (Gabon).

(c) Julien Bonhomme 
Fig.3.

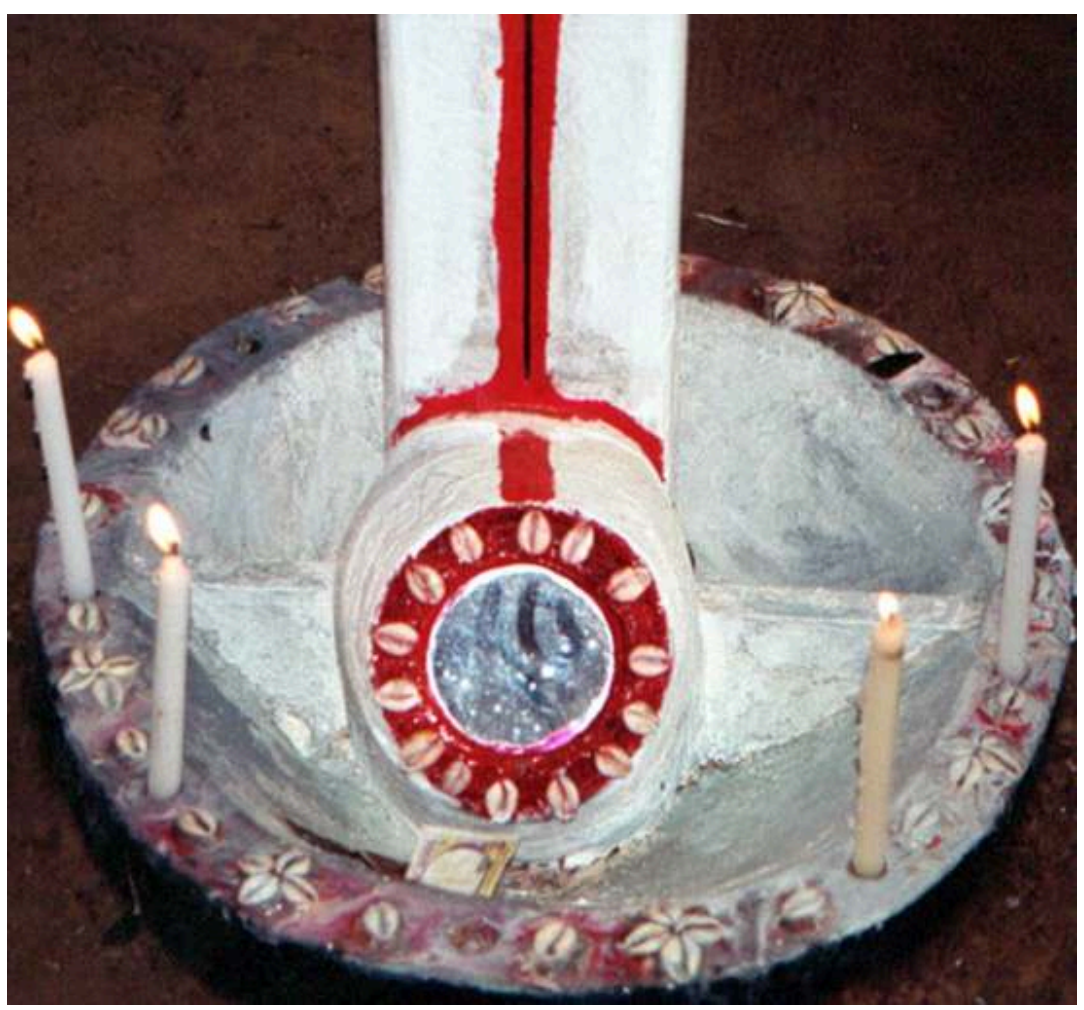

Base du poteau central d'un temple de Bwete (Gabon).

(c) Julien Bonhomme

Dans toute l'Afrique centrale, le champ sémantique de la vision sature le vocabulaire de la divination : le devin est un clairvoyant. Le miroir constitue alors un instrument de choix pour mettre en scène cette clairvoyance. Dans l'aire kongo, des miroirs sont souvent incrustés sur le ventre des fétiches nkisi (fig. 1). Ils dissimulent les substances magiques placées dans le ventre du fétiche, tout en figurant le monde invisible et la clairvoyance du devin (dont ils sont probablement les supports). Les statuettes fang du Gabon possèdent de même des yeux en laiton, verre ou miroir ${ }^{22}$. Les fétiches du Bwete corporation initiatique du Gabon dont nous allons reparler - incorporent eux aussi des miroirs (fig. 2 et 3). Ces miroirs divinatoires sont souvent spécifiquement employés à la découverte des voleurs et des sorciers. Chez les Gikuyu du Kenya, le contre-sorcier peut faire apparaître dans un miroir le visage d'un voleur dont il "découpe » ensuite le reflet à l'aide d'un rasoir afin de le tuer ${ }^{23}$. Dans toute une partie de l'Afrique, le miroir est ainsi devenu l'outil privilégié de nombre des mouvements anti-sorcellerie du $\mathrm{XX}^{\mathrm{e}}$ siècle. Dans la Rhodésie du Nord (actuelle Zambie) des années 1930, les witch-finders du mouvement Bamucapi pratiquent une nouvelle épreuve de détection des sorciers: « hommes et femmes sont alignés sur deux files et doivent passer un par un dans le dos $\mathrm{du}$ witch-finder qui, par un mouvement du poignet, capture leur reflet dans un petit miroir circulaire. Ce reflet est supposé permettre de détecter sur le champ les sorciers $»^{24}$. Le même rituel se retrouve dans la Tanzanie des années 1950 avec le mouvement des Wacilole, littéralement « les gens du miroir », mais aussi en pays yoruba au Nigeria. Le miroir constitue ainsi le nouvel instrument divinatoire d'un rituel résolument lié à la modernité coloniale, comme en témoignent les élégants habits 
européens portés par les devins Bamucapi ou l'inspection des suspects qui miment une revue militaire.

Fig.4.

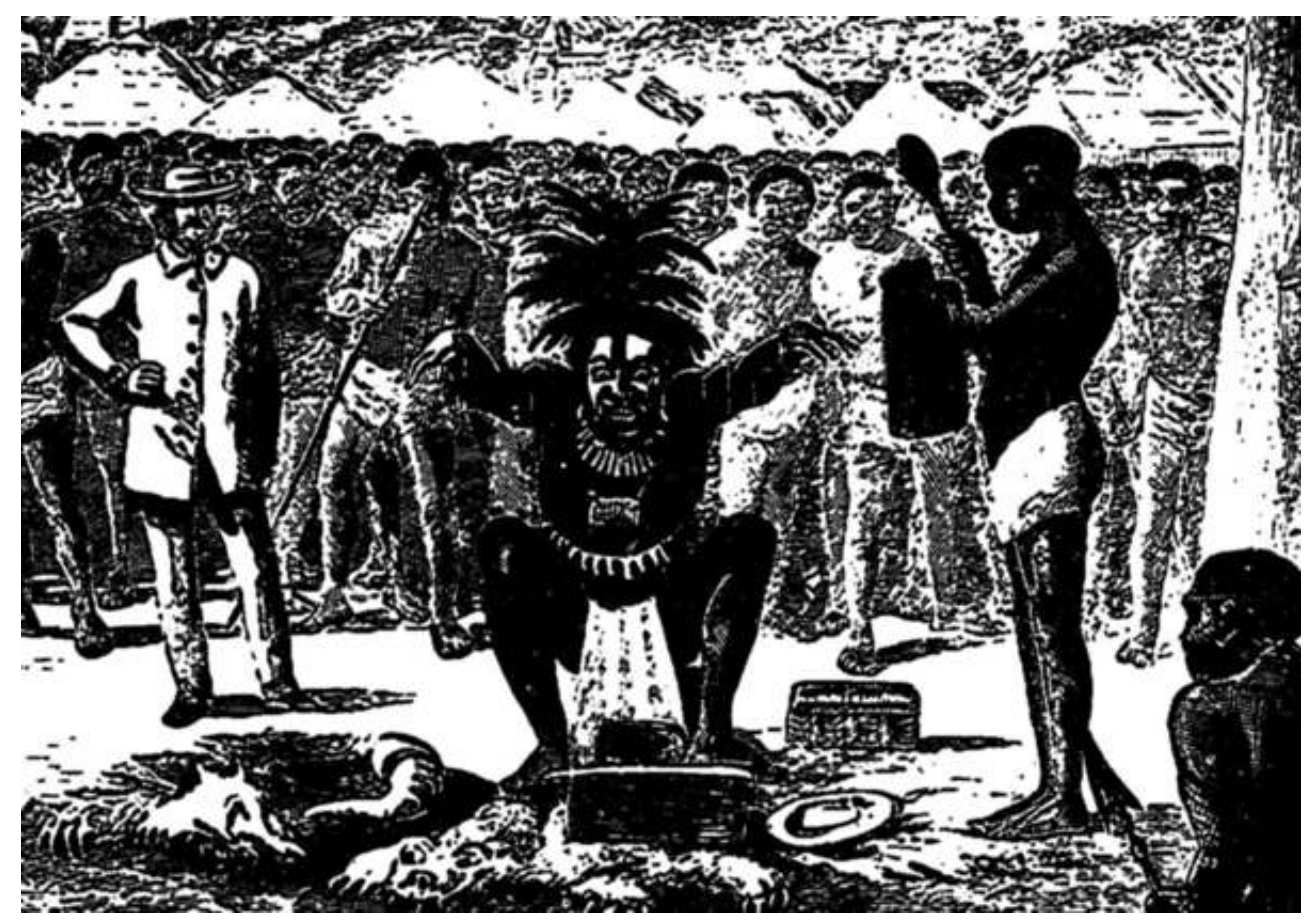

Gravure originale, divination par le miroir (Gabon). Dans Paul Belloni du Chaillu, Voyages et aventures en Afrique équatoriale, Paris, Michel Lévy Frères, 1867, p. 270.

$14 \mathrm{Au}$ Gabon, les miroirs divinatoires sont attestés depuis longtemps. En 1857, l'explorateur Paul du Chaillu assiste chez les nkomi à une séance de divination par le miroir : « [le devin] s'assit sur un escabeau puis déposa devant lui un nouveau coffret, rempli de talismans et surmonté d'un miroir [...]. Jambuai fut invité à nommer successivement et à haute voix tous les habitants; à chaque nom, le devin consultait son miroir, pour savoir si la personne nommée n'était pas le sorcier qu'il cherchait » $(\text { fig. } 4)^{25}$. Au début du XXe siècle, les Pygmées emploient eux aussi un miroir divinatoire pour démasquer les sorciers ou retrouver un objet perdu ou voléer. Ces miroirs d'importation se sont substitués aux anciens supports de la divination par le reflet pratiquée depuis fort longtemps: cuivre poli et surtout surface de l'eau. Du Chaillu observe chez les gisira de l'hinterland qu'« une cruche de terre noire, remplie d'eau, et entourée de craie magique et de fétiches, remplaçait les miroirs en usage chez les tribus de la côte ${ }^{27}$. Un mythe d'origine de la divination raconte d'ailleurs comment un homme trouve en forêt l'arbuste eboga ( Tabernanthe iboga) dont il déterre les racines pour les ramener au village. La nuit venue, il s'assied au corps de garde et mange les racines hallucinogènes. Dehors, il pleut. Une flaque se forme à l'entrée du corps de garde. L'homme contemple le reflet de la lune dans l'eau et y découvre tous les secrets du village. Une énigme initiatique, reprenant ce mythe, affirme que le premier miroir fut le reflet de la lune dans l'eau. Et les devins du "Misoko de l'assiette » préfèrent aujourd'hui encore utiliser la surface de l'eau plutôt que le miroir (fig. 5). 
Fig.5.

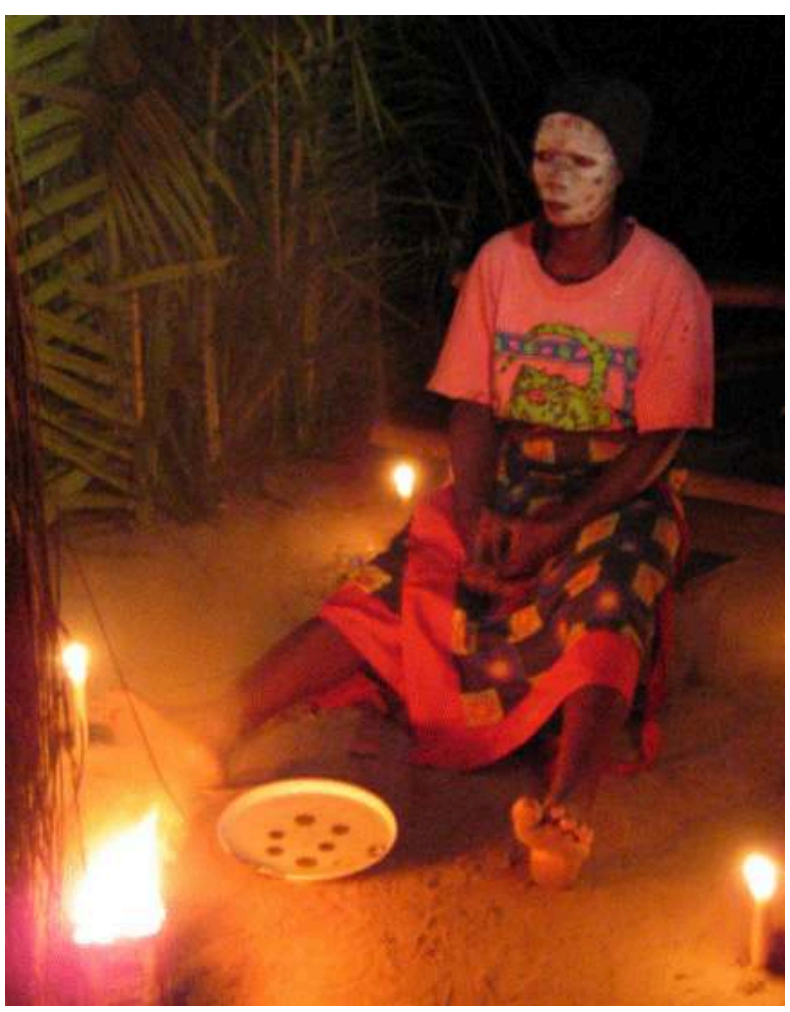

Divination du « Misoko de l'assiette » (Gabon).

(c) Patrick Mauroy

15 Le miroir est ainsi devenu l'accessoire emblématique des nganga-a-Misoko, les devins de la corporation initiatique du Bwete Misoko (de sokogo, "découvrir ") ${ }^{28}$. Chaque nganga possède un petit miroir portatif (parfois collé sur une corne fétiche) auquel un traitement rituel a conféré un pouvoir divinatoire pour peu que soient respectées certaines précautions d'usage : exposé à la lumière du soleil, il ne révèle plus rien ; ayant reflété des rapports sexuels, il se ternit ; aucune femme ne doit le regarder quand elle a ses règles. Lors d'une consultation divinatoire, le nganga scrute alternativement son miroir et le patient en face de lui (fig. 6, 7 et 8). Il est censé y voir défiler la vie et les raisons de l'infortune de ce dernier. Le patient est en effet venu dans l'espoir que le devin identifiera dans le miroir le parent sorcier qu'il suspecte toujours être à l'origine de son malheur. 
Fig.6.

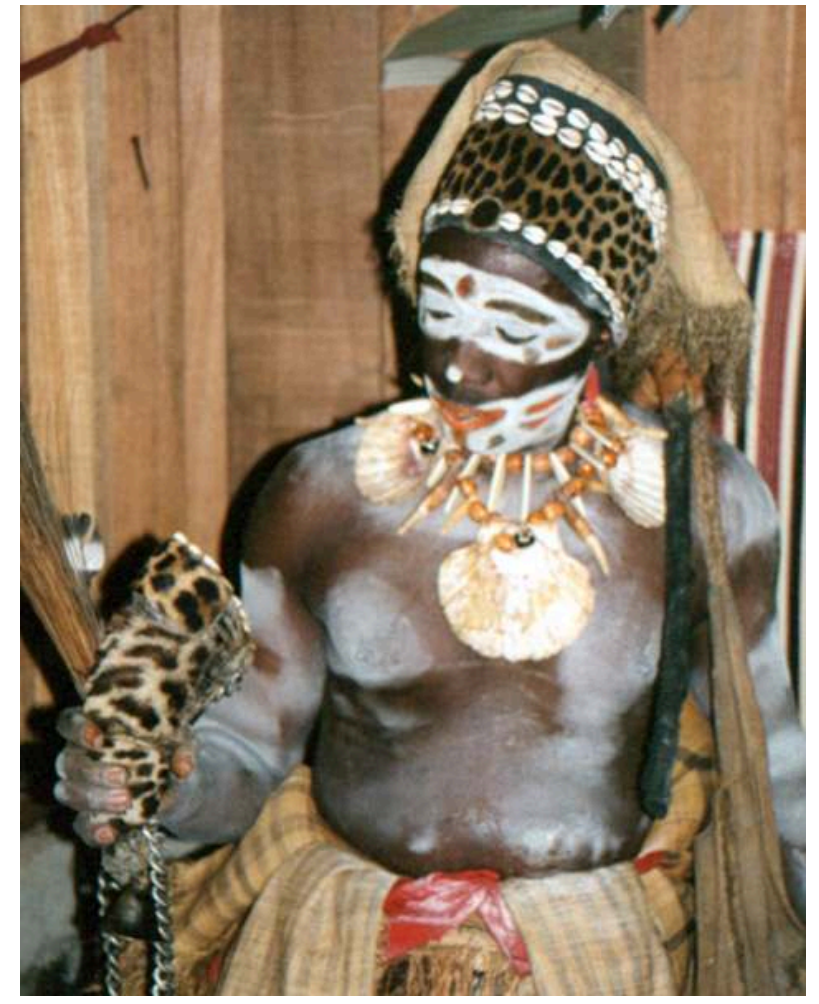

Divination par le miroir (Gabon)

(C) Julien Bonhomme 
Fig.7.

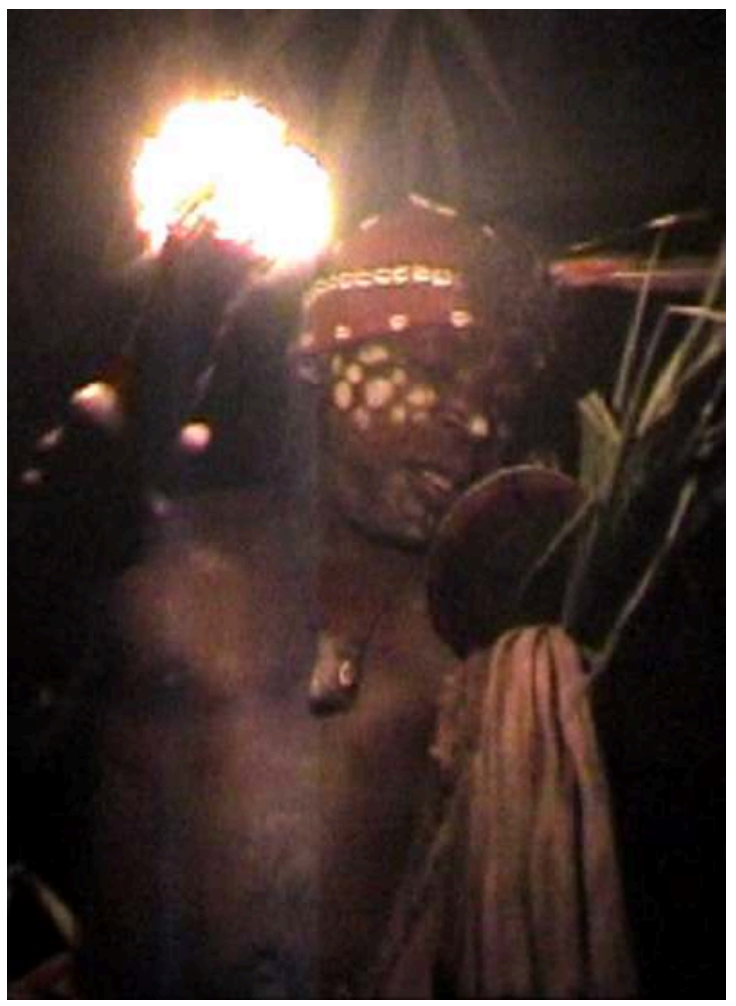

Divination par le miroir (Gabon).

(C) Julien Bonhomme

Fig.8.

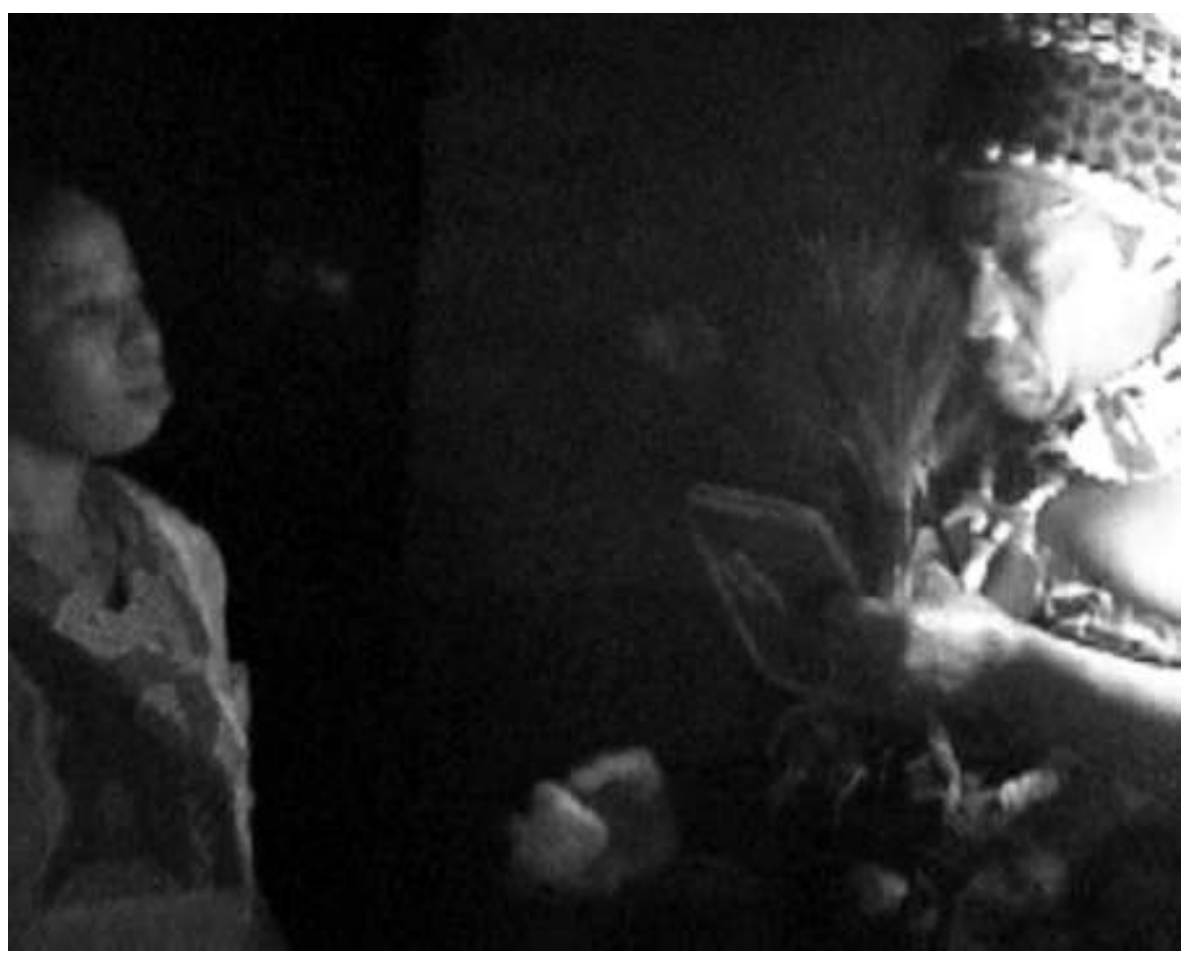

Divination par le miroir (Gabon).

(C) Julien Bonhomme 
Tous ces usages divinatoires reposent ainsi sur le pouvoir révélateur d'un miroir qui capte l'invisible ${ }^{29}$. En Afrique centrale, cet invisible renvoie à la sorcellerie, c'est-à-dire à la part occulte des relations sociales, notamment des relations entre parents. En effet, ce sont avant tout les sorciers et leurs auxiliaires maléfiques qui se trouvent reflétés dans les miroirs. La morale publique repose sur l'évitement du conflit: en public, les dissensions doivent être esquivées par une hypocrisie policée permettant de sauver les apparences de la solidarité entre parents. C'est pourquoi les sorciers agissent toujours de nuit ou dans le dos des gens, dérogeant à la norme de la visibilité réciproque dans les interactions de face-à-face ${ }^{30}$. C'est en cela que la sorcellerie relève de l'invisible. On comprend alors l'intérêt du miroir : à l'image du devin Bamucapi scrutant le reflet des suspects défilant derrière lui, la divination par le miroir est une rétrovision qui permet de voir par-derrière, c'est-à-dire de rendre visible l'invisible sorcellaire. Cette perception indirecte, médiation subtile entre confrontation en face-à-face et dissimulation de dos, révèle les gens tels qu'ils sont en vérité : sous la figure de sorciers. Une histoire édifiante racontée par un nganga gabonais illustre bien ce pouvoir révélateur du reflet: si l'on dispose un miroir devant le sexe d'une femme endormie, son reflet la fait voir sous l'aspect d'un démon cornu - preuve, selon mon interlocuteur, que toutes les femmes sont des sorcières en puissance!

17 Le dispositif rituel du miroir divinatoire permet en définitive de réfléchir l'image révélatrice d'autrui. Dans la divination du Bwete Misoko, ce jeu spéculaire est en fait plus complexe puisque images de soi et d'autrui se mêlent. En effet, le nganga qui scrute alternativement son patient et son petit miroir ne voit en réalité dans ce dernier que son propre reflet. Certains devins sont bien prêts à l'avouer : «On ne voit rien dans le miroir. On ne voit que soi-même. C'est ta figure, bien sûr. Mais c'est le Bwete . C'est pourquoi on dit que le Bwete, c'est toi-même ». Le va-et-vient entre le patient et le reflet de soi doit en outre permettre de faire surgir l'image mentale du sorcier. Le miroir divinatoire fait ainsi apparaître de multiples images qui se réfléchissent les unes dans les autres: le devin, le patient, le sorcier et même l'étrange Bwete. Cette réflexivité spéculaire généralisée se retrouve dans certains rites initiatiques d'Afrique centrale qui mobilisent le miroir de manière encore plus élaborée.

\section{Miroir initiatique : réfléchir les ancêtres}


Fig.9.

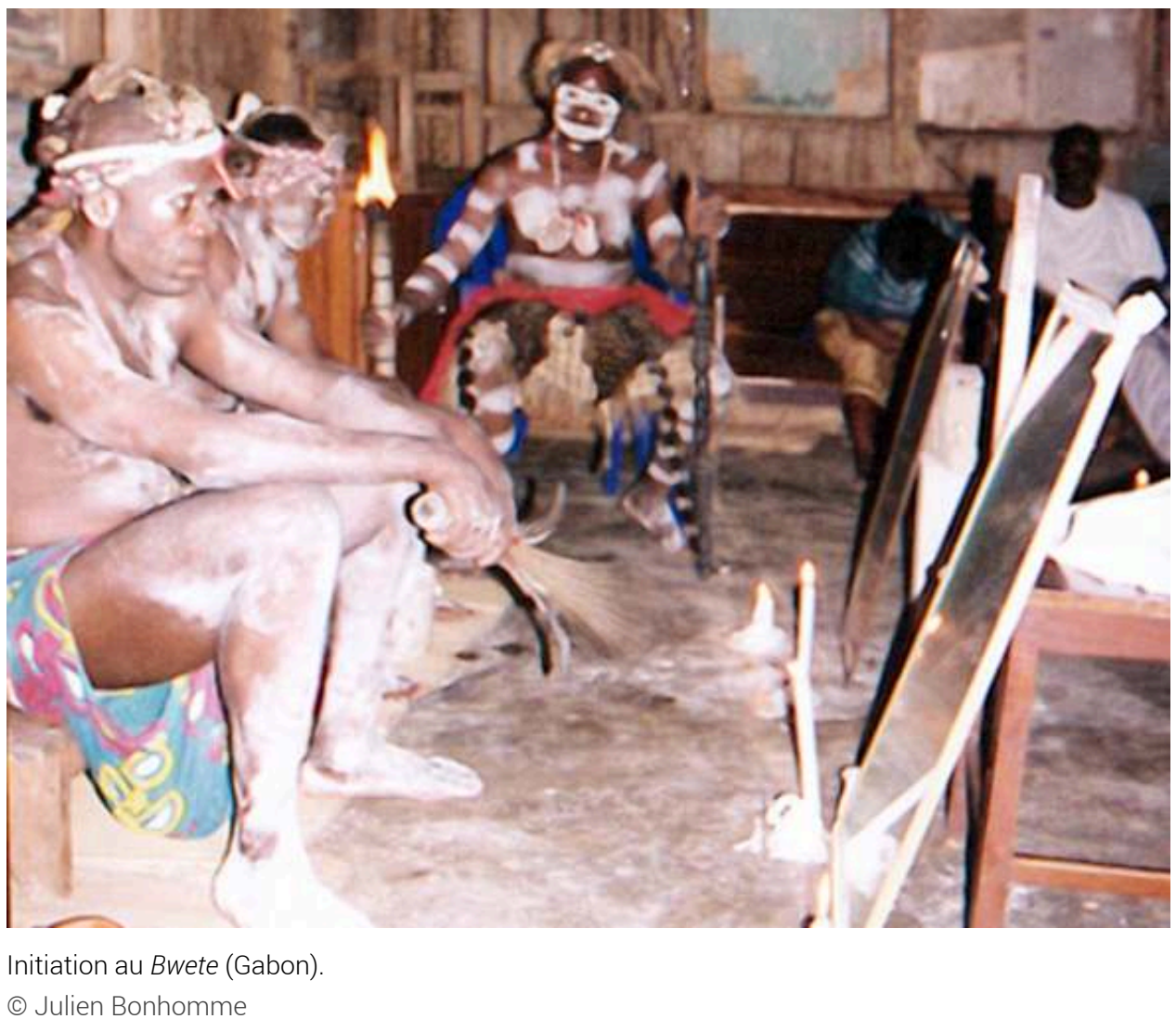

18 La corporation initiatique du Bwete Misoko emploie le miroir aussi bien dans ses rites de passage que dans la consultation divinatoire. Lors de l'initiation, le néophyte banzi, gavé de racines hallucinogènes $d^{\prime}$ eboga, est assis toute une nuit en face d'un grand miroir en pied qu'il doit fixer sans détourner le regard (fig. 9). Sous les effets conjugués de l' eboga et de la focalisation sur le miroir, l'initié a des visions qu'il doit raconter au fur et à mesure aux initiateurs assis à ses côtés (l'hallucinogène ne provoquant pas de perte de conscience). Ce dispositif initiatique exploite les deux paradoxes du miroir. D'une part, le paradoxe de l'espace spéculaire, cet « impossible espace que peuplent les reflets ${ }^{31}$, permet de représenter le monde du Bwete où sont supposées se dérouler les scènes visionnaires ${ }^{32}$. D'autre part, le paradoxe du reflet spéculaire permet un dédoublement de l'initié : assis devant le miroir, il se trouve également au-delà du miroir, dans le monde du Bwete. Ce double visuel est gedina-dina, l'âme-ombre ou âmereflet qui accompagne tout vivant ${ }^{33}$. Restant conscient, le néophyte n'est pas un corps inerte que son esprit aurait temporairement quitté. De là la complexité du sujet visionnaire par rapport au sujet ordinaire pour lequel les instances de la vision et de l'action sont confondues et univoques. Qui du double ou de l'initié perçoit les visions? Tantôt l'initié devant le miroir assiste en spectateur extérieur à des scènes dans le miroir. Tantôt il est le témoin de scènes qui s'adressent directement à lui : il est alors happé dans le miroir, son propre point de vue devenant intérieur à la scène. Sous l'effet de cette mise en abyme de la perception visuelle, c'est alors le double qui devient spectateur et acteur dans le monde du Bwete, se distinguant ainsi de l'initié immobile devant le miroir. Cette distinction se brouille cependant lorsque l'initié se met à mimer devant le miroir les actions que son double accomplit dans le miroir (pagayer, ouvrir un 
paquet fétiche, frapper un sorcier, etc.). Cette ambiguïté spéculaire, que renforce la confusion due à l' eboga, permet d'instaurer un rapport équivoque d'identification et de distinction entre l'initié et son double. La situation est déconcertante au point que les néophytes, souvent plus perplexes que crédules, hésitent quant au poids ontologique à attribuer à leur propre expérience initiatique.

D'un point de vue pragmatique, le dispositif rituel du miroir opère ainsi une très efficace redéfinition des indexicaux ( «je», «ici», et dans une certaine mesure " maintenant») et, par là même, instaure un contexte d'interaction et d'énonciation tout à fait distinct des situations ordinaires ${ }^{34}$. Le miroir confère en effet un espace et un sujet à des visions qui risqueraient autrement de n'être qu'hallucinations. Cette mise en abyme spéculaire est décisive. Le dédoublement et l'inclusion de l'initié dans son propre champ de vision sont en effet les conditions minimales de la transformation initiatique. Le face-à-face concentré du néophyte avec son propre reflet manifeste que l'initiation est un retour sur soi : le banzi se focalise sur lui-même et sur les problèmes qui l'ont fait venir manger l' eboga. Fixer intensément sa propre image - et notamment se regarder soi-même dans les yeux - permet ainsi d'instaurer une relation avec soi (fig. 10). Mais loin de se réduire à une simple tautologie, cette réflexivité spéculaire ouvre sur une série d'écarts ambigus. Le retour sur soi est en effet toujours un travail de transformation. La concentration obstinée sur le reflet dépouille le visage de sa familiarité jusqu'à le faire devenir autre. L'initié voit par exemple son visage se métamorphoser en celui d'un pygmée, d'un gorille, d'un vieillard. L'image du miroir est ainsi un écran de projection, le visage représentant le schéma de base à partir duquel se forment des visions de plus en plus élaborées ${ }^{35}$. Ce schéma du visage est d'autant plus saillant que la face blanche de l'initié badigeonné de kaolin se détache nettement sur le fond noir du reflet (l'initiation se déroulant dans l'obscurité). 
Fig.10.

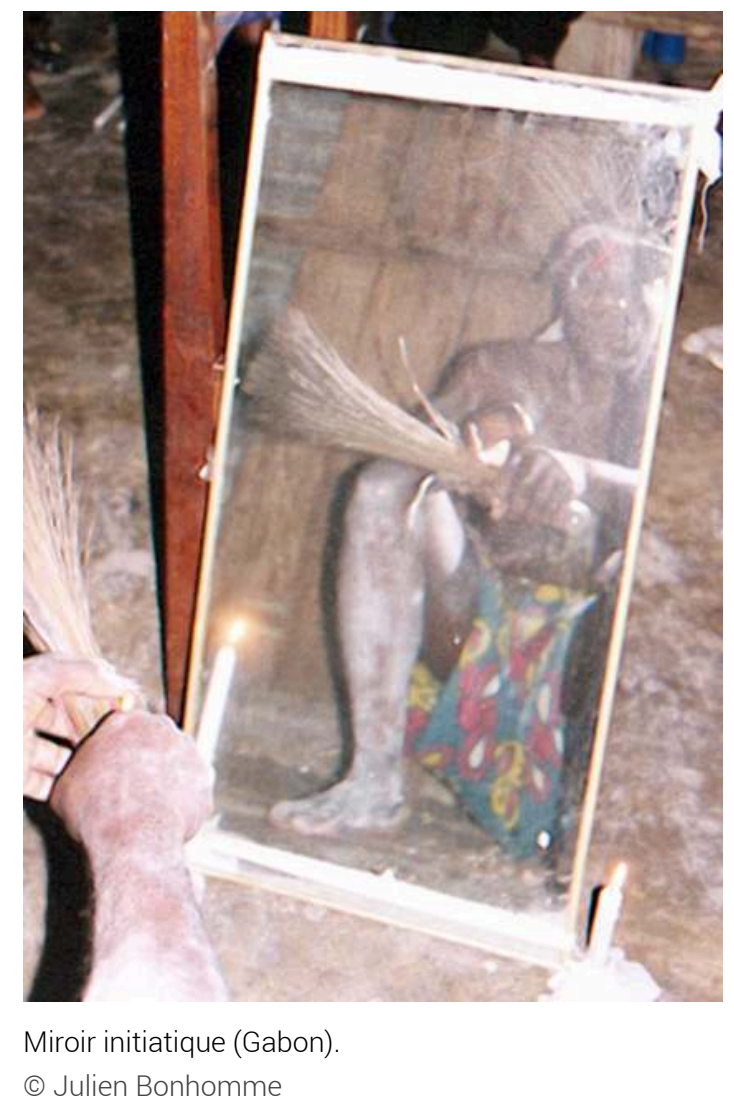

20 Les métamorphoses de la face spectrale du banzi font apparaître de multiples personnages : parents, animaux, fantômes avec lesquels le double spéculaire va pouvoir interagir. La relation spéculaire n'est donc pas solipsiste. L'un des personnages clefs de ces rencontres visionnaires est le parent sorcier, responsable supposé du malheur ${ }^{36}$. Contrairement au recours divinatoire, l'initiation repose ainsi sur un usage personnel du miroir qui permet un face-à-face visionnaire de l'infortuné avec un sorcier jusqu'à présent invisible. Ce même usage du miroir se retrouve dans la plupart des initiations thérapeutiques au Gabon: Ombudi des mitsogo du centre du pays, Mbiri des fang au Nord, Ombwiri des myene à l'Ouest, Mabanzi des gisira et bapunu au Sud ${ }^{37}$. Dans tous ces cas, la découverte spéculaire du sorcier ou du génie afflicteur constitue une étape décisive du processus de résolution de l'infortune.

Mais le sorcier n'est pas le seul personnage que rencontre l'initié dans le miroir. Plus importante encore est la vision du Bwete, figure générique des ancêtres protecteurs qui peut revêtir diverses apparences. Cette apparition, qui survient habituellement vers la fin du voyage visionnaire, provoque une intense jubilation. Si le sorcier joue un rôle prépondérant dans le Misoko (à visée thérapeutique), l'ancêtre est le protagoniste principal du Disumba, branche originelle du Bwete à visée plus religieuse. Ancêtre et sorcier représentent ainsi les deux pôles opposés entre lesquels s'inscrivent tous les autres personnages des visions (parents, animaux, Pygmées, Sirènes, Blancs) selon qu'ils sont plutôt en position d'adjuvants ou d'opposants (fig. 11). Cette image spéculaire de l'ancêtre constitue la révélation centrale de la société initiatique. En Afrique centrale, le monde des morts est souvent aquatique : la cosmologie kongo s'organise ainsi autour d'une symétrie en miroir entre le monde des morts sous l'eau et 
celui des vivants à la surface ${ }^{38}$. Les morts sont les reflets énantiomorphes des vivants dans un miroir aquatique - de là les inversions dont ils sont coutumiers (ils ont les pieds inversés, font tout à l'envers, etc.). Par l'entremise du miroir, le rite initiatique met donc en scène la relation spéculaire entre les ancêtres et les vivants.

Fig. 11.

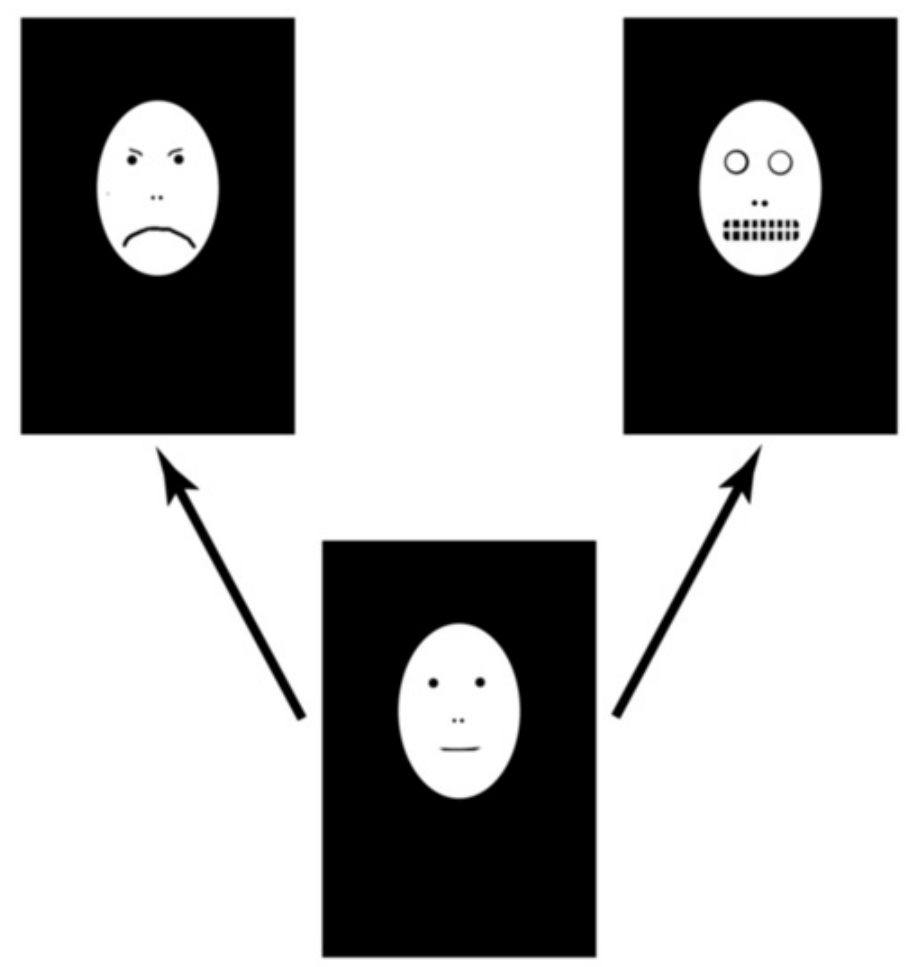

Métamorphoses initiatiques du visage en sorcier et en ancêtre.

L'association entre ancêtre et miroir peut être plus directe encore. Le miroir que scrutent les néophytes est parfois fixé sur l'abdomen de la statuette surplombant le panier reliquaire contenant les ossements ancestraux: «Le Bwiti [...] consiste matériellement en un bâton grossièrement sculpté en figure humaine; des éclats de verre figurent les yeux ; un autre morceau de miroir occupe la place du nombril : c'est là que le sorcier cherchera la vérité. Au-dessous, une peau de chat-tigre, quelques clochettes, des herbes, des lanières, etc.; puis, dans un sac d'où sort ce bâton sculpté, une tête de mort remplie de poudres diverses, de sanie de cadavre, et d'os de serpent ${ }^{39}$. Cette association se retrouve de manière plus spectaculaire encore dans le "miracle des crânes» de l'initiation au Melan, rite de passage du Byeri, culte des ancêtres chez les fang du Gabon et du Cameroun. Le néophyte gavé de l'hallucinogène alan ( Alchornea floribunda) est assis dos aux crânes lignagers face à un miroir qu'il doit fixer. Puis, par un pivotement du miroir, les initiateurs font apparaître les crânes à la place de son propre visage ${ }^{40}$. Le pivotement permet de mettre astucieusement en relation la figure du néophyte et celle de l'ancêtre.

De semblables subterfuges catoptriques fondés sur l'oblicité ou le pivotement se retrouvent dans des contextes religieux hors d'Afrique. Dans un temple bouddhique au Japon, un miroir oblique fait apparaitre l'image d'un dieu ${ }^{41}$. De même, un miroir est enchâssé dans un mur du sanctuaire de Déspoina à Lycosoura en $\operatorname{Arcadie}^{42}$. Celui qui s'y 
regarde ne distingue qu'une image obscure de lui-même. En revanche, les dieux y apparaissent " en pleine clarté ", sans doute grâce à l'inclinaison du miroir qui reflète les statues placées au centre du temple. Selon la théorie optique des Grecs, le miroir fait apparaitre l' eidolon ${ }^{43}$. Alors qu'elle deviendra simple artifice imitatif à l'époque classique, dans la Grèce archaïque, cette image est une entité fantomatique qui voisine avec fantômes (phasma), spectres (psuche) et autres apparitions oniriques (oneiros). En reflétant une image spectrale du sujet, le miroir de Lycosoura ouvre donc sur les ténèbres d'Hadès. Mais en reflétant l'éclat des dieux, le même miroir devient par ailleurs l'instrument d'une véritable théophanie optique. Autre exemple de mise en scène catoptrique, ne curieuse fresque de la Villa Item (dite Villa des Mystères) à Pompéi figure un satyre scrutant le fond d'une coupe à boire tandis qu'un autre satyre brandit derrière lui un masque de silène grimaçant (fig. 12). Il est possible que cette peinture évoque une scène de divination relevant des rites dionysiaques ${ }^{44}$. Cela suggère que miroir et masque ont pu être associés dans des mises en scène au cours des Mystères dionysiaques, à l'image du crâne et du miroir du Byeri gabonais.

Fig. 12 .

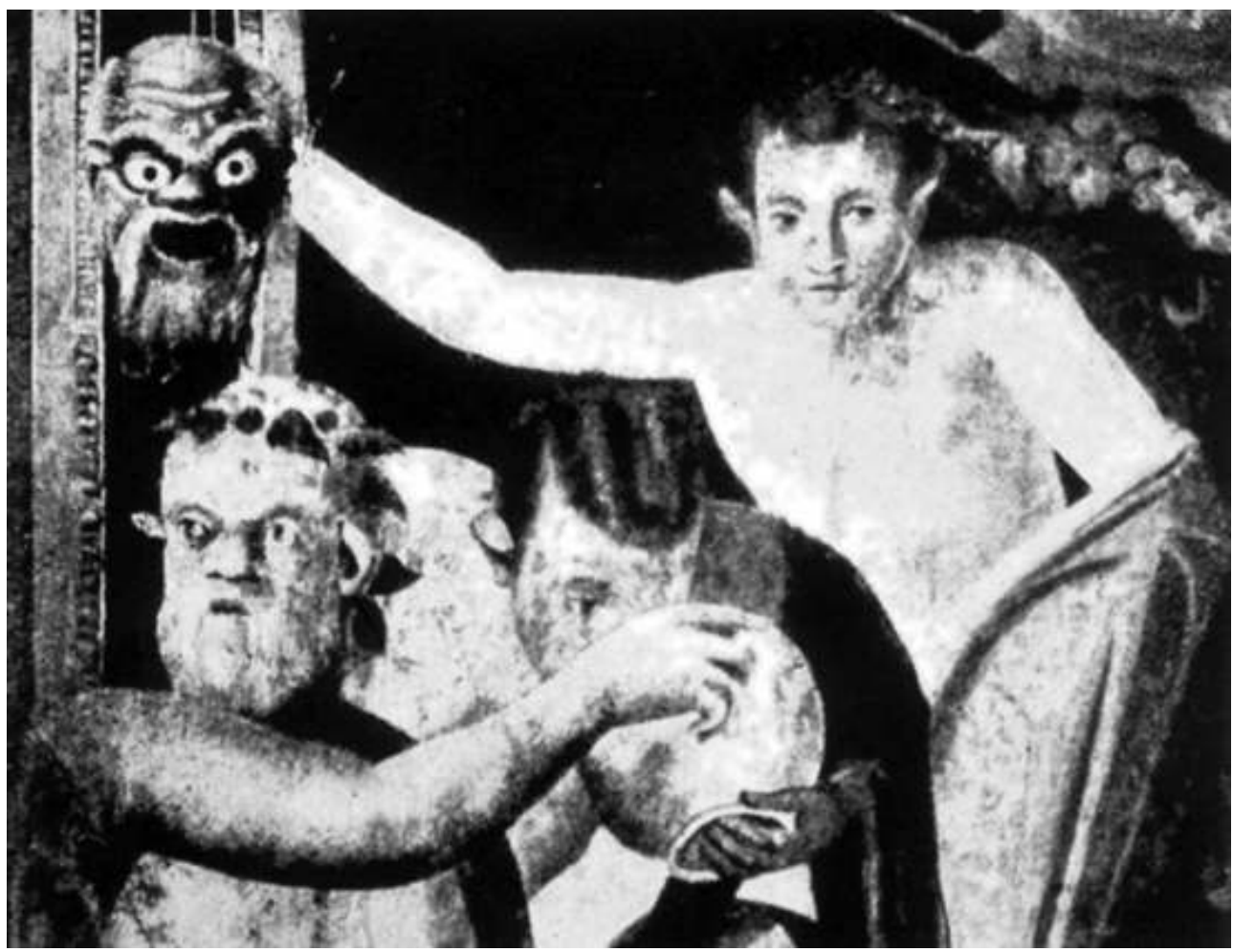

Détail de fresque, Villa dite des « Mystères » (ou Villa Item), scène de catoptromancie (Pompei). Dans Armand Delatte, La catoptromancie grecque et ses dérivés, Paris, Droz, 1932, fig. 21.

Miracle des crânes d'Afrique centrale, théophanie bouddhique ou grecque, toutes ces manipulations magico-religieuses du miroir illustrent que le rituel n'est pas un système symbolique abstrait mais d'abord et avant tout un ensemble d'interactions concrètes. Les manipulations «symboliques » du rituel sont en effet adossées à des manipulations matérielles, perceptives et relationnelles. De là l'importance des objets : ils possèdent une valeur opératoire décisive car ils sont au centre des mises en scène sur lesquelles se fonde l'efficacité propre du rituel. Les choix qui président à leur sélection obéissent donc à des critères de pertinence. Le rituel privilégie en effet ce qu'on pourrait appeler 
des "objets paradoxaux", objets sélectionnés pour leur capacité à engendrer des paradoxes lorsqu'on les manipule. Le masque, qui tout à la fois dissimule et donne à voir, est sans doute le plus répandu d'entre eux, tant il est présent dans nombre de cérémonies initiatiques ou religieuses de par le monde. Mais le miroir, ce véritable «instrument du paradoxe ${ }^{45}$, en est assurément un autre. Exploitant l'étrangeté cognitive de l'expérience spéculaire, les usages rituels du miroir permettent en effet des manipulations paradoxales de l'identité. Cela en fait de très efficaces supports instrumentaux pour des rites initiatiques qui visent à mettre le sujet en relation avec divers agents (Dieu, esprits, ancêtres). L'oscillation du reflet entre identité familière et altérité étrange rend ainsi possible une identification paradoxale des initiés au Bwete et aux ancêtres, relation qui prend parfois un tour ouvertement ironique lorsque les initiateurs révèlent que l'on ne voit évidemment rien d'autre que son propre reflet dans le miroir et que le Bwete, c'est en définitive soi-même.

\section{Miroirs analogiques : réfléchir ego et ses alter}

Dans l'un de ses articles, U. Eco se pose la question de savoir si le reflet est un signe ${ }^{46}$. $\mathrm{Au}$ terme de son analyse, il conclut que l'image spéculaire est a-sémiotique, sauf dans les cas de miroirs déformants ou de mises en scène catoptriques. Dans ces cas seulement, le reflet devient un signe, au sens scolastique du terme : aliquid pro aliquo, quelque chose qui vaut pour quelque autre chose. Les mises en scène rituelles du miroir précédemment évoquées reposent sur un tel emploi du reflet comme signe. Il s'agit toujours de faire apparaître une image à la place d'une autre image. Ces usages rituels se situent ainsi au point d'articulation entre perceptif et sémiotique. Ils ancrent en effet leur portée symbolique sur une propriété optique du miroir : sa capacité à produire des images étranges. Le pouvoir réfléchissant du miroir opère une mise en abyme d'ego et focalise l'attention sur son image. Cette image n'est toutefois pas tautologique. Le reflet creuse toujours un écart au coeur de la ressemblance. Le reflet énantiomorphe du visage d'ego donne en réalité à voir le visage d'un alter ego : partie invisible du corps, notre visage ne peut en effet faire l'objet d'aucune perception visuelle directe, contrairement au visage d'autrui. Cette inquiétante étrangeté du reflet est encore renforcée par les manipulations du miroir : inclinaison ou pivotement permettent de faire apparaître de nouvelles images, de les mettre en relation, de les transformer les unes dans les autres. Les rituels spéculaires rendent ainsi possible un travail sur l'identité à travers une mise en relation du visage d'ego avec une série d'autres figures.

En définitive, le miroir rituel est toujours un « miroir anormal » : on y voit autrui plutôt que soi-même. Cette conclusion conduit à relativiser l'association classique du miroir et du narcissisme. Dans la littérature romantique, l'importance du miroir, et plus généralement du double, possède indéniablement une dimension narcissique fortement teintée d'angoisse ${ }^{47}$ : ainsi chez Jean-Paul ( Siebenkäs, 1797), Chamisso (L'homme qui a perdu son ombre, 1813), Hoffmann (op. cit.), Dostoïevski (Le Double, 1846) ou Wilde ( Le portrait de Dorian Gray, 1890). On ne peut suivre en revanche Géza Róheim qui rapporte les représentations et les usages les plus divers du miroir à une fixation narcissique, régression à la seconde étape de l'ontogenèse psychosexuelle ${ }^{48}$. En Afrique centrale, les usages rituels du miroir ne sont pas narcissiques : on ne s'y abîme pas dans l'image de soi, mais au contraire dans celle d'autrui. Les initiés du Bwete qui se regardent si attentivement dans leur miroir pour se maquiller au début d'une cérémonie ne sont pas 
amoureux de leur image : ils scrutent plutôt leur transformation en autre chose qu'euxmêmes. Maquillés et accoutrés, ils deviennent en effet des mikuku, c'est-à-dire des esprits ancestraux.

27 Ces miroirs rituels sont donc des miroirs analogiques : un visage apparait à la place d'un autre visage, aliquid pro aliquo. Le reflet se met à diverger, la réflexion se fait diffraction. C'est pourquoi le miroir possède une pertinence singulière dans les cultures organisées autour d'une cosmologie analogique ${ }^{49}$. Afin d'éclairer ce point mais aussi de mettre en perspective une comparaison plusieurs fois esquissée, quittons l'Afrique pour l'Europe afin de faire ressortir l'importance du miroir dans la cosmologie chrétienne. Selon le mythe grec, Dionysos se regarde dans un miroir au moment où les Titans le mettent à mort. Le reflet redouble le démembrement : « en se reflétant dans le miroir, Dionysos se voue à la multiplicité $»^{50}$. Le néo-platonisme reprend le mythe pour insérer la polymorphie dionysiaque dans une cosmologie analogique. Instrument de l'anagogie vers l'Un, le miroir se voit alors conférer une dimension métaphysique qui perdure jusque dans le christianisme ${ }^{51}$. Ce dernier manifeste cependant une profonde ambivalence à l'égard du miroir comme à l'égard des images. A partir d'une matrice platonicienne qui fait osciller l'image entre apparence trompeuse et reflet analogique de l'Idée, le christianisme n'a de cesse d'opposer un mauvais et un bon miroir : miroir de fausseté versus miroir de vérité ${ }^{52}$. L'opposition correspond en réalité à un mauvais et un bon usage de l'analogisme, en fonction de l'image de référence à laquelle toutes les autres images sont rapportées : miroir du Diable versus miroir de Dieu. Le miroir est diabolique dans la mesure où il constitue un pouvoir de mystification. Le Diable est luimême un speculum fallax. Un dicton médiéval affirme d'ailleurs que «le miroir est le vray cul du diable » (fig. 13). L'Église condamne par conséquent la catoptromancie, mantique marquée du signe du Malin. Mais elle interdit également les miroirs dans les couvents de peur d'exciter la concupiscence des yeux: mieux vaut y placer les Écritures, ce miroir divin. La Bible est en effet un "miroir sans tâche", speculum sine macula (Sagesse VII, 27) car elle reflète la perfection divine. Le christianisme médiéval fait ainsi du miroir un symbole de la remontée anagogique vers Dieu. Les livres de compilation des connaissances sont eux-mêmes des miroirs: Speculum mundi ou Speculum majus qui invitent à la « spéculation » (speculatio), contemplation de la gloire de Dieu reflétée par le miroir des créatures ${ }^{53}$. 
Fig.13.

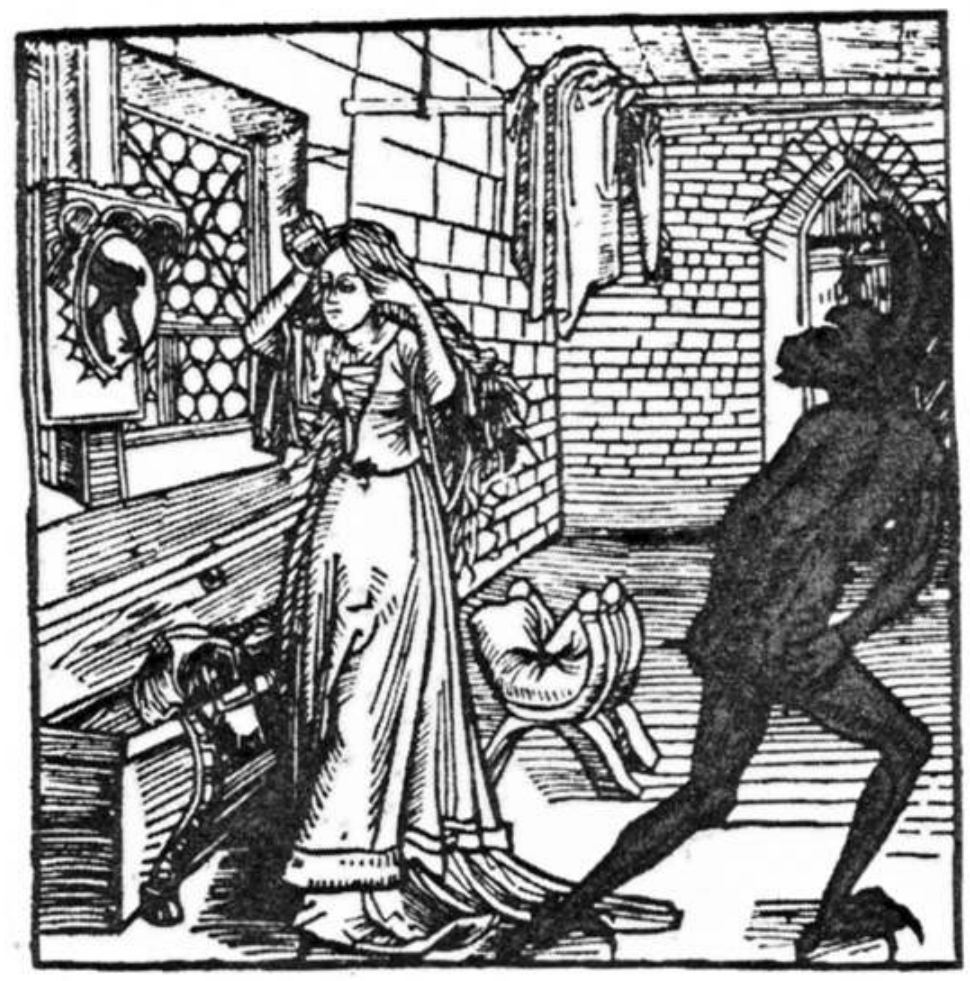

Gravure originale, le diable dans le miroir. Dans Der Ritter vom Turn, Bâle, 1493 publié dans Jurgis Baltrusaitis, Le miroir. Révélations, science-fiction et fallacies, Paris, Le Seuil-Aline Elmayan, 1978, p. 192.

$\mathrm{Au}$ XVII ${ }^{\mathrm{e}}$ siècle, l'Europe baroque se passionne pour les miroirs qui permettent de faire jouer les vertiges de l'analogie. C'est l'époque des curiosités catoptriques: anamorphoses à miroir, machines et maisonnettes catoptriques, théâtres de Protée, jardins et pays d'illusion ${ }^{54}$. En 1646 parait à Rome l' Ars magna lucis et umbrae d'Athanase Kircher, inventeur de la lanterne magique et maître ès métamorphoses. On peut y voir le Speculum multiplex qui fait apparaître une tête à visages innombrables, le Theatrum polydicticum qui réfléchit une scénette à l'infini, le Speculum heterodicticum qui fait défiler à la place du visage du spectateur la tête d'un âne, d'un boeuf, d'un cerf ou d'un oiseau de proie, ou encore le Speculum multividium qui fait apparaître une double tête à triple oeil, image de la Sainte Trinité (fig. 14). Les noms baroques de ces machines catoptriques témoignent à eux seuls d'un analogisme qui s'adonne aux délices de la multiplication infinie des apparences. L'exemple du Speculum multividium prouve toutefois que ces "aberrations optiques" restent ordonnées dans une cosmologie chrétienne. L'analogisme baroque se boucle sur Dieu et ses miroirs reflètent encore une image divine. Comme l'écrit Gaspard Schott dans sa Magia universalis naturae et artis (en 1659) : Speculum est Christus . 
Fig.14.

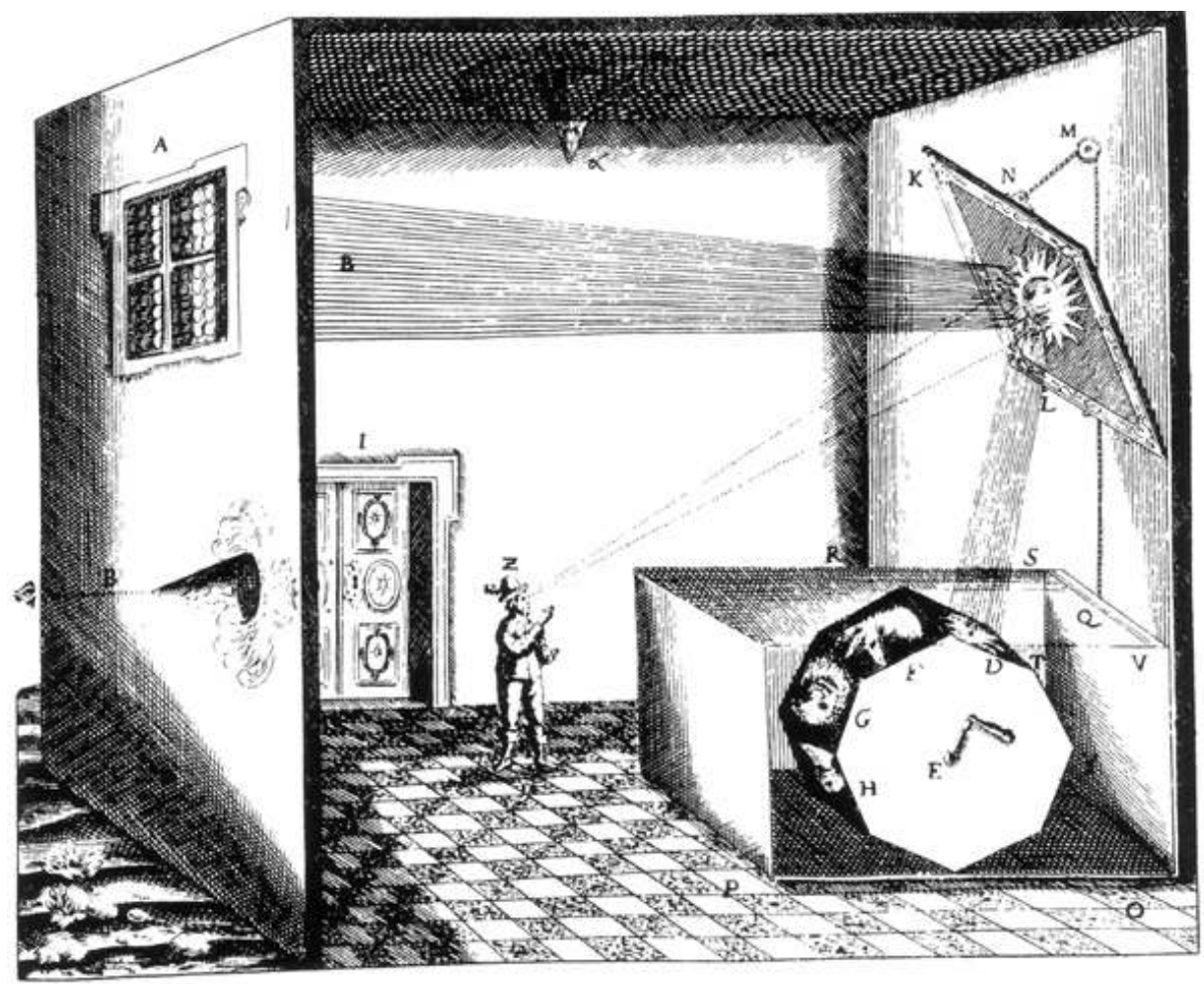

Machine changeant les hommes en animaux. Dans Athanasius Kircher, Ars magna lucis et umbrae, Rome, 1646, publié dans Jurgis Baltrusaitis, Le miroir. Révélations, science-fiction et fallacies, Paris, Le Seuil-Aline Elmayan, 1978, p. 45.

Pour résumer cela de manière quelque peu cavalière, on pourrait dire que dans un miroir, les Chrétiens voient tantôt Dieu tantôt le Diable alors que les Africains y voient tantôt les ancêtres tantôt les sorciers. Dans les deux cas, l'image du sujet se définit par rapport à une série d'autres images, série ordonnée autour d'une image de référence. Le miroir analogique transforme le reflet d'ego en l'image de son $"$ significant other $\aleph^{55}$, cette altérité constituante à partir de laquelle se définit le sujet. Étant donné l'ambivalence du miroir, les images de référence sont en réalité doubles: l'une bonne (Dieu, l'ancêtre) qui fonde l'identité d'ego, l'autre mauvaise (le Diable, le sorcier) qui menace son intégrité. Il y a en somme un terme organisateur et un terme désorganisateur. A différents types de société, différents types de significant other et donc différents types d'image spéculaire.

Quand une société se transforme, le reflet de ses miroirs se transforme alors avec elle. Les reflets d'ancêtres et de parents sorciers sont en effet typiques des sociétés segmentaires africaines structurées par la parenté. La centralisation politique des royaumes africains fait alors apparaître de nouvelles images: le roi remplace les ancêtres du lignage. Au royaume de Loango, le Nganga Nyambi scrute son miroir divinatoire pour découvrir qui sera le prochain souverain Maloango : il le voit apparaître à l'image de Dieu $^{56}$. Roi du Dahomey de 1858 à 1889, Glèlè aménage dans son palais une "entrée des glaces" afin que son pouvoir s'y reflète à l'infini. Agbidinukum, descendant et historien du roi, commente : «Glèlè a dit qu'avec cette entrée, il donnait un exemple au peuple du Dahomey. Il a dit que c'est le roi Glèlè qui est le miroir, et tout le monde doit le regarder pour voir clairement le Dahomey». Il ajoute: «Si l'on 
regarde dans l'eau, on se voit. Glèlè est le reflet. Quand on regarde, on le voit toujours $\aleph^{57}$. L'entrée des glaces constitue une magnification optique du pouvoir de Glèlè : ses sujets voient la figure royale dans leur propre reflet démultiplié. Son palais ressemble ainsi à la galerie des glaces du palais de Versailles, mise en scène de la monarchie absolue.

Dans l'Europe moderne, le miroir a servi de support instrumental à une autre transformation de longue durée. Lewis Mumford a bien mis en valeur le rôle du miroir, dont la diffusion se généralise dans la seconde moitié du XVII siècle à la faveur de nouvelles techniques de production, dans l'avènement de l'individualisme bourgeois ${ }^{58}$. La banalisation des miroirs contribue à la naissance de l'autoportrait comme genre pictural, de même qu'elle popularise l'introspection, accompagnant ainsi l'émergence d'un sujet singulier doté d'une conscience de soi. Dans le miroir, le reflet de Dieu laisse lentement la place au reflet de l'individu, dont on pourrait dire qu'il est le significant other de la modernité occidentale ${ }^{59}$. C'est dans ce contexte historique de transition entre créature de Dieu et individu moderne qu'a lieu la confrontation coloniale, point de rencontre entre le miroir européen et le miroir africain.

Fig. 15.

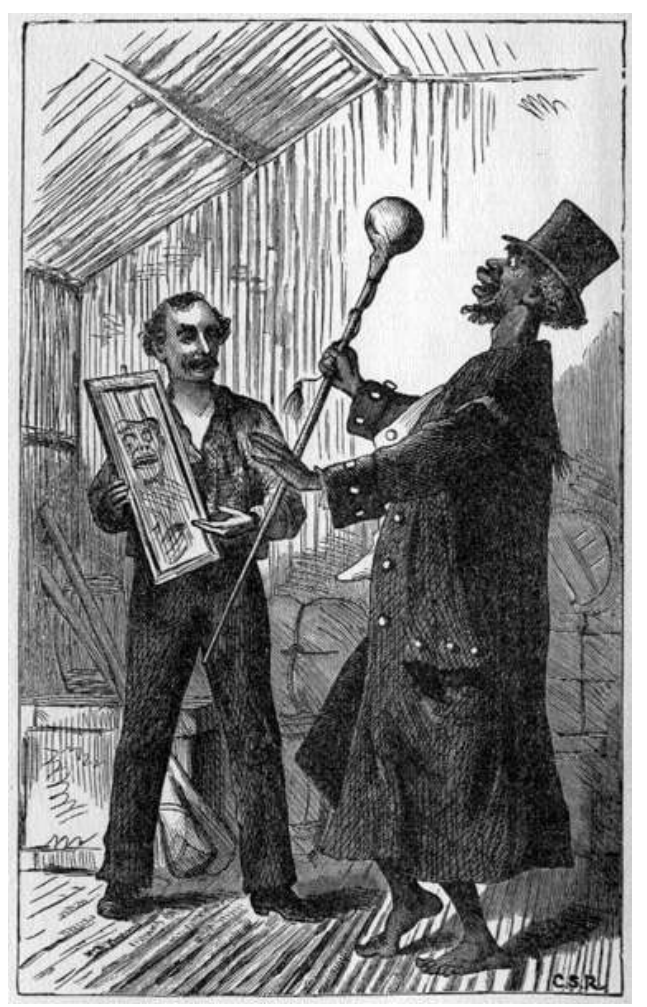

Gravure originale, Du Chaillu et le chef Quenguéza. Dans Paul Belloni du Chaillu, The country of the dwarfs, New York, Harper, 1871, gravure hors-texte. 
Fig.16.

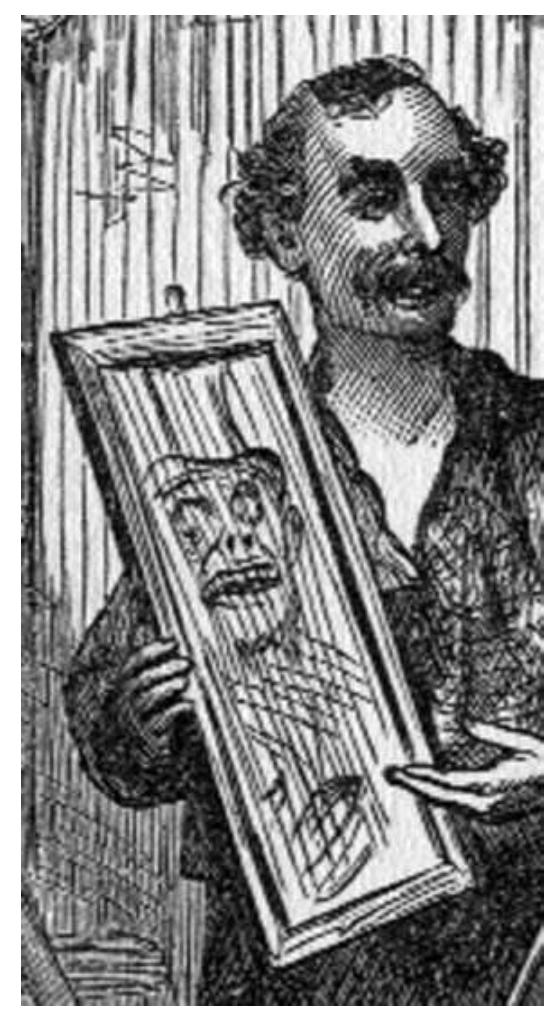

Gravure originale, Du Chaillu et le chef Quenguéza. Dans Paul Belloni du Chaillu, The country of the dwarfs, New York, Harper, 1871, gravure hors-texte.

Les Européens ont mis en scène la rencontre coloniale pour en faire un théâtre enchanté visant à impressionner les autochtones par une manifestation aussi magique que technique de leur puissance - ce qui fait dire à J. Fabian que l'impérialisme fut une entreprise théâtrale ${ }^{60}$. A côté des gramophones, aimants et autres piles électriques, le miroir a joué un rôle de premier plan dans les premiers contacts entre Africains et explorateur européens. Certes, le cliché de l'indigène découvrant stupéfait son reflet dans un miroir tendu par l'explorateur est sans aucun doute une mystification : qui ne s'est pas déjà miré dans un reflet d'eau? Ce n'est cependant pas son propre visage mais un reflet bien plus étrange que l'autochtone découvre dans le miroir colonial : l'image de soi en train de devenir autre. Jean et John Comaroff ont souligné l'importance de l'usage missionnaire du miroir : les missionnaires tendent aux autochtones un miroir afin qu'ils puissent s'y découvrir créatures de Dieu - d'où l'ambivalence de ces derniers à l'égard de cet objet qui menace de dérober leur identitée ${ }^{61}$. Le miroir civilisateur fait ainsi activement partie du projet colonial qui vise à policer des "sauvages " pour en faire des sujets. Lorsque au Gabon, Paul du Chaillu offre une redingote et un haut-deforme au chef Quenguéza, il lui tend ensuite une glace afin de lui donner à voir une image de lui-même en sujet civilisé ${ }^{62}$. La gravure qui accompagne la description ne peut toutefois s'empêcher de dépeindre un Quenguéza grotesque, allant jusqu'à le doter d'un reflet simiesque, sans doute pour souligner le chemin que les «sauvages" doivent encore accomplir sur la voie de la civilisation (fig. 15 et 16). Le miroir met ainsi en abyme la rencontre coloniale en en réfléchissant les effets. S'y croisent les reflets ambivalents de chacun dans le miroir de l'autre. Si dans le miroir de l'explorateur, le reflet de l'indigène hésite entre le singe et le gentleman, dans le miroir des nganga 
apparaissent bientôt des spectres blancs, tantôt pris pour des ancêtres, tantôt pour des mauvais génies.

\section{NOTES}

1. Ainsi, mirabilia, « merveille » ou miratio, «étonnement ».

2. Jack Goody, Representations and contradictions: ambivalence towards images, theatre, fiction, relics, and sexuality, Oxford, Blackwell Publishers, 1997.

3. Geneviève Sennequier (éd.), Miroirs. Jeux et reflets depuis l'Antiquité, Paris, Somogy-Éditions d'art, 2000.

4. Corpus ethnographique principal de l'article, les données concernant les usages du miroir au Gabon proviennent d'un travail de terrain mené depuis 2000 sur les rites initiatiques et divinatoires, notamment dans le centre et le sud du pays.

5. Olfert Dapper, Description de l'Afrique, Amsterdam, Wolfgang, Waesberge, Boom \& van Someren, 1686, p. 328.

6. Elikia M'Bokolo, Noirs et blancs en Afrique équatoriale, Paris, EHESS, 1981, p. 108.

7. Joseph Tonda, Le Souverain moderne. Le corps du pouvoir en Afrique centrale (Congo, Gabon), Paris, Karthala, 2005, p. 78-79.

8. Cf. notamment Julius von Negelein, «Bild, Spiegel und Schatten im Volksglauben ", in Archiv für Religionswissenschaft, vol.5, n¹, 1902, p.1-37. Géza Róheim, Spiegelzauber, Leipzig, Internationaler Psychoanalytischer Verlag, 1919.

9. James G. Frazer, Le Rameau d'Or. Tome 1. Le Roi magicien dans la société primitive. Tabou et les périls de l'âme [1911], Paris, Robert Laffont, 1981.

10. Germaine Dieterlen (dir.), La notion de personne en Afrique noire, Paris, CNRS, 1973.

11. Joseph Tonda, op. cit., p. 76-78.

12. René Zazzo, Reflets de miroir et autres doubles, Paris, PUF, 1993. Anne-Marie Fontaine, L'enfant et son image, Paris, Nathan, 1992. Pierre Mounoud, Annie Vinter (dir.), La reconnaissance de son image chez l'enfant et l'animal, Neuchâtel, Delachaux \& Niestlé, 1981.

13. Le visage est en effet partie invisible de notre corps : l'enfant n'apprend donc jamais à re connaître son visage dans le miroir mais seulement à le connaître (contrairement au visage d'autrui).

14. Jacques Postel, «Troubles de la reconnaissance spéculaire de soi au cours des démences tardives ", in J. Corraze (éd.), Image spéculaire du corps, Toulouse, Privat, 1980, p. 215-271.

15. Sigmund Freud, «L'inquiétante étrangeté » [1919], in L'inquiétante étrangeté et autres essais, Paris, Gallimard, 1985, p. 257.

16. Pour reprendre la belle expression de Jorge L. Borges dans L'auteur et autres textes, Paris, Gallimard, 1965, p. 29.

17. Jacques Lacan, «Le stade du miroir comme formateur de la fonction du Je (1949) », in Écrits, Paris, Gallimard, 1966, p. 90.

18. Au Gabon: Julien Bonhomme, Le miroir et le crâne. Parcours initiatique du Bwete Misoko (Gabon), Paris, CNRS, 2005. Au Congo: Christiane Falgayrettes-Leveau (dir.), Le Geste kôngo, Paris, Dapper, 2002. En RDC : Zoe S. Strother, «Smells and bells: the role of skepticism in Pende divination ", in J. Pemberton III (ed.), Insight and Artistry in African Divination, Washington, Smithsonian Institution Press, 2000, p. 99-115. En Angola : Manuel Jórdan, « Art and divination 
among the Chokwe, Lunda, Luvale, and other related peoples of Northwestern Zambia ", in J. Pemberton III, op. cit., p. 134-143. Au Malawi, en Zambie et au Zimbabwe : Audrey I. Richards, "A modern movement of witch-finders " [1935], repris dans M. Marwick, Witchcraft and Sorcery, Harmondsworth, Penguin Books, 1970, p. 164-177. En Tanzanie : Roy G. Willis, « The Kamcape : an anti-sorcery movement in south-west Tanzania », in Africa, vol. 38, $\mathrm{n}^{\circ} 1,1968$, p. 1-15. Au Nigeria : P. Morton-Williams, « The Atinga Cult among the South-Western Yoruba : A sociological analysis of a witch-finding movement ", in Bulletin de l'institut français d'Afrique noire, vol. 18, n³-4, 1956, p. 315-334. Au Kenya : Michel Adam. « Nouvelles considérations dubitatives sur la théorie de la magie et de la sorcellerie en Afrique noire ", in L'Homme, ${ }^{\circ} 177-178,2006$, p. 279-302.

19. Caroline Humphrey \& Urgunge Onon, Shamans \& elders : experience, knowledge, and power among the Daur Mongols, Oxford, Clarendon Press, 1996.

20. Dominique Gallois, « O pajé waiãpi e seus "espelhos" », in Revista de antropologia, vol. 27-28, 1984-1985, p. 179-196. Pour un intéressant récit mythique yanomami incluant des miroirs, cf. Bruce Albert \& David Kopenawa (éds.), Yanomami : L'esprit de la forêt, Arles, Actes Sud, 2003.

21. Armand Delatte, La catoptromancie grecque et ses dérivés, Paris, Droz, 1932.

22. Philippe Laburthe-Tolra, Fang, Paris, Dapper, 1991.

23. Michel Adam, op. cit., p. 294.

24. Audrey I. Richards, op. cit., p. 164-165 (je traduis).

25. Paul B. du Chaillu, Voyages et aventures en Afrique équatoriale, Paris, Michel Lévy Frères, 1867, p. 271.

26. Henri Trilles, Les pygmées de la forêt équatoriale, Paris, Bloud \& Gay, 1932, p. 174-181.

27. Paul B. du Chaillu, L'Afrique sauvage, Paris, Michel Lévy Frères, 1868, p. 149. Le même usage divinatoire du " pot à médecines » rempli d'eau se retrouve chez les azande du Soudan (Edgar E. Evans-Pritchard, Sorcellerie, oracles et magie chez les Azandé [1937], Paris, Gallimard, 1972, p. 209).

28. Le Bwete (ou Bwiti ) est un rite initiatique originaire des mitsogo du Gabon central mais aujourd'hui largement répandu, notamment dans la moitié sud du pays. Le Bwete Misoko, sa branche divinatoire et thérapeutique, est ainsi pratiqué par la plupart des ethnies du Gabon. Le Bwete remonte au-delà du XIXe siècle, puisque les premiers explorateurs l'ont trouvé déjà bien implanté dans l'hinterland gabonais.

29. Chez les mitsogo, miroir se dit d'ailleurs geèno, du verbe ènaga, « voir ».

30. Julien Bonhomme, "Voir par-derrière. Sorcellerie, initiation et perception au Gabon ", in Social Anthropology, vol. 13, n³, 2005, p. 259-273.

31. Pour reprendre une autre belle expression de Jorge L. Borges (op. cit., p. 121).

32. La mydriase induite par l'eboga modifie la perception de la profondeur de champ, rendant ainsi encore plus étrange l'espace spéculaire.

33. Terme construit par redoublement augmentatif à partir du substantif gedina, "ombre», « image », " photographie ».

34. Sur le contexte pragmatique du rituel, cf. Carlo Severi, « Memory, reflexivity and belief. Reflections on the ritual use of language ", in Social Anthropology, vol. 10, n¹, 2002, p. 23-40.

35. Les initiés comparent d'ailleurs l'écran du miroir au cinéma ou à la télévision.

36. L'initiation au Bwete Misoko est invariablement motivée par une suspicion de sorcellerie déclenchée par une répétition du malheur.

37. Sur l' Ombudi : Otto Gollnhofer, Roger Sillans, «Phénoménologie de la possession chez les Mitsogho (Gabon). Rites et techniques", in Anthropos, vol. 74, n5-6, 1979, p. 737-752. Sur le Mbiri : James W. Fernandez, Bwiti. An ethnography of the religious imagination in Africa, Princeton, Princeton University Press, 1982, p. 595-599.

38. Wyatt Mac Gaffey, Religion and Society in Central Africa. The BaKongo of Lower Zaire, Chicago, The University of Chicago Press, 1986, p. 43. 
39. Alexandre Le Roy, La Religion des Primitifs, Paris, Beauchesne, 1911, p. 348-349. Voir aussi André Raponda-Walker, Roger Sillans, Rites et croyances des peuples du Gabon, Paris, Présence Africaine, 1962, p. 204.

40. James W. Fernandez, Bwiti, op. cit., p. 264.

41. Jurgis Baltrusaitis, Le miroir. Révélations, science-fiction et fallacies, Paris, Le Seuil-Aline Elmayan, 1978, p. 170.

42. Jean-Pierre Vernant, «Au miroir de Méduse », in L'individu, la mort, l'amour. Soi-même et l'autre en Grèce ancienne, Paris, Gallimard, 1996.

43. Jean-Pierre Vernant, Figures, idoles, masques, Paris, Julliard, 1990. Gérard Simon, Le Regard, l'être et l'apparence dans l'Optique de l'Antiquité, Paris, Seuil, 1988.

44. Armand Delatte, op. cit., p. 189-202. P. Veyne soutient cependant que la scène n'est en réalité qu'un «enfantillage» au cours d'un banal «matin de noces au gynécée »: un satyre farceur brandit un masque pour effrayer la femme représentée sur un pan perpendiculaire de la fresque (Paul Veyne, «La fresque dite des Mystères à Pompéi ", in Paul Veyne, François Lissarrague, Françoise Frontisi-Ducroux, Les mystères du gynécée . Paris, Gallimard, 1998, p. 13-153). On peut néanmoins objecter que le regard du satyre et son masque ne sont pas tournés vers ladite femme mais bien dans l'axe de l'autre satyre et de la coupe qui lui est tendue (et dont il scrute visiblement le fond d'un oeil concentré).

45. James W. Fernandez, «Reflections on looking into mirrors ", in Semiotica, vol. 30, n¹-2, 1980, p. 36 .

46. Umberto Eco, «Sugli specchi », in Sugli specchi e altri saggi, Milano, Bompiani, 1985, p. 9-37.

47. Otto Rank, Don Juan et Le Double [1932], Paris, Payot, 1973.

48. Geza Róheim, op. cit., p. 260-263.

49. Pour Philippe Descola, l'analogisme désigne un mode d'identification des existants reposant sur un réseau de correspondances entre singularités disparates (Philippe Descola, Par-delà nature et culture, Paris, Gallimard, 2005, p. 280-320).

50. Jean-Pierre Vernant, Figures, idoles, masques, op. cit., p. 135.

51. Jean Pépin, "Plotin et le miroir de Dionysos", in Revue internationale de philosophie, vol. 24, n92, 1970, p. 304-320.

52. Jurgis Baltrusaitis, op. cit.

53. Marie-Hélène Tesnière, « Des livres appelés Miroirs ", in G. Sennequier, op. cit., p. 124-127.

54. Jurgis Baltrusaitis, op. cit.

55. James W. Fernandez, «Reflections on looking into mirrors », op. cit .

56. R.E. Dennett, At the back of the black man's mind [1906], London, Frank Cass \& co, 1968, p. 30 et p. 51 .

57. Suzanne Preston-Blier, «Le Roi Glèlè du Dahomey. Les portraits divinatoires d'un roi lion et homme de fer », in C. Falgayrettes-Leveau (éd.), "Magies", Paris, Dapper, 1996, p. 134.

58. Lewis Mumford, Technics and Civilization, New York, Harcourt, Brace \& World, 1934, p. 128-131.

59. C'est pourquoi le miroir narcissique ne peut en réalité être que moderne.

60. Johannes Fabian, Out of our minds: reason and madness in the exploration of Central Africa, Berkeley, University of California Press, 2000, p. 121.

61. Jean Comaroff, John Comaroff, of Revelation and Revolution. Vol. 1. Christianity, Colonialism, and Consciousness in South Africa, Chicago, University of Chicago Press, 1991, p. 185-189.

62. Paul B. du Chaillu, L'Afrique sauvage, op. cit., p. 27. 


\section{RÉSUMÉS}

Cet article s'intéresse aux usages divinatoires et initiatiques du miroir en Afrique centrale, et notamment au Gabon. Il montre que le miroir constitue un instrument de choix pour opérer des manipulations rituelles paradoxales aussi concrètes qu'efficaces. Son pouvoir réfléchissant permet une mise en abyme du sujet, tandis que sa manipulation permet une mise en rapport avec une série d'autres personnes. Le miroir rend ainsi possible un travail complexe sur l'identité à travers une mise en relation ambiguë du soi et de l'autre. L'article met alors en perspective le rôle instrumental du miroir pour des conceptions analogiques du sujet, avant de conclure sur l'importance des mises en scène spéculaires dans la rencontre coloniale entre l'Afrique et l'Europe.

\section{INDEX}

Index chronologique : XXe siècle

Index géographique : Afrique centrale, Gabon

Thèmes : anthropologie

Mots-clés : altérité, divination, fantôme, image, miroir, rituel

\section{AUTEUR}

\section{JULIEN BONHOMME}

Julien Bonhomme est Maître de conférences en anthropologie à l'Université Lumière Lyon-2 et mène des recherches de terrain au Gabon, entre autres sur les rituels initiatiques et la sorcellerie. Il est notamment l'auteur d'un livre paru aux éditions du CNRS en 2005 : Le Miroir et le Crâne. Parcours initiatique du Bwete Misoko (Gabon).

julienbonhomme@yahoo.fr 